\title{
Lipopolysaccharide and Curcumin Co-Stimulation Potentiates Olfactory Ensheathing Cell Phagocytosis Via Enhancing Their Activation
}

\author{
Ding-Jun Hao ${ }^{1,2}$. Cuicui Liu ${ }^{1,3}$ - Lingling Zhang ${ }^{1,3}$ - Bo Chen ${ }^{1,3}$ • Qian Zhang ${ }^{1,3}$. \\ Rui Zhang ${ }^{1,3}$ • Jing An ${ }^{1,3}$ • Jingjing Zhao ${ }^{1,3}$ - Mingmei $\mathrm{Wu}^{4}$ - Yi Wang ${ }^{1,3}$. \\ Alfred Simental $^{5}$ - Baorong He $\mathrm{H}^{1,2} \cdot$ Hao Yang ${ }^{1,3}$
}

Published online: 14 October 2016

(C) The American Society for Experimental NeuroTherapeutics, Inc. 2016

\begin{abstract}
The gradual deterioration following central nervous system (CNS) injuries or neurodegenerative disorders is usually accompanied by infiltration of degenerated and apoptotic neural tissue debris. A rapid and efficient clearance of these deteriorated cell products is of pivotal importance in creating a permissive environment for regeneration of those damaged neurons. Our recent report revealed that the phagocytic activity of olfactory ensheathing cells (OECs) can make a substantial contribution to neuronal growth in such a hostile environment. However, little is known about how to further increase the ability of OECs in phagocytosing deleterious products. Here, we used an in vitro model of primary cells to investigate the effects of lipopolysaccharide (LPS) and curcumin (CCM)
\end{abstract}

Electronic supplementary material The online version of this article (doi:10.1007/s13311-016-0485-8) contains supplementary material, which is available to authorized users.

Ding-Jun Hao

haodingjun@126.com

$\checkmark$ Baorong He

hebr888@163.com

$\checkmark$ Hao Yang

yanghao.71_99@yahoo.com

1 Shaanxi Spine Medicine Research Center, Hong Hui Hospital, Xi'an Jiaotong University College of Medicine, Shaanxi, China

2 Department of Spine Surgery, Hong Hui Hospital, Xi'an Jiaotong University College of Medicine, Shaanxi 710054, China

3 Translational Medicine Center, Hong Hui Hospital, Xi'an Jiaotong University College of Medicine, Shaanxi 710054, China

4 Institute of Neurosciences, The Fourth Military Medical University, Shaanxi 710032, China

5 Department of Otolaryngology-Head and Neck Surgery, Loma Linda University Medical Center, Loma Linda, CA 92354, USA co-stimulation on phagocytic activity of OECs and the possible underlying mechanisms. Our results showed that costimulation using LPS and CCM can significantly enhance the activation of OECs, displaying a remarkable upregulation in chemokine (C-X-C motif) ligand 1 , chemokine (C-X-C motif) ligand 2, tumor necrosis factor- $\alpha$, and Toll-like receptor 4 , increased OEC proliferative activity, and improved phagocytic capacity compared with normal and LPS- or CCM-treated OECs. More importantly, this potentiated phagocytosis activity greatly facilitated neuronal growth under hostile culture conditions. Moreover, the up-regulation of transglutaminase-2 and phosphatidylserine receptor in OECs activated by LPS and CCM co-stimulation are likely responsible for mechanisms underlying the observed cellular events, because cystamine (a specific inhibitor of transglutaminase-2) and neutrophil elastase (a cleavage enzyme of phosphatidylserine receptor) can effectively abrogate all the positive effects of OECs, including phagocytic capacity and promotive effects on neuronal growth. This study provides an alternative strategy for the repair of traumatic nerve injury and neurologic diseases with the application of OECs in combination with LPS and CCM.

Keywords Olfactory ensheathing cells $\cdot$ Cell activation . Lipopolysaccharide $\cdot$ Curcumin $\cdot$ Phagocytosis $\cdot$ Neuron growth

\section{Introduction}

Olfactory ensheathing cells (OECs) have hitherto been regarded as a leading potential candidate in cell transplantation therapies for nerve repair. Compelling evidence indicates that OECs exhibit a range of valuable cell attributes, including 1) active stimulation of neuronal survival and axon 
regeneration by secretion of numerous neurotrophic growth factors, neurite-promoting guidance molecules, and physical substrates; and 2) critical aspects of tissue repair by structural remodeling and support, enhancement of antigenic stimuli, immune phagocytosis, and penetration of astrocytic glial scar tissue [1-11]. Based on these unique properties, OECs could be a special cell type with a strong comprehensive potential for proregeneration. Nevertheless, an increasing number of controversial issues related to the proregeneration capabilities of OECs are debated, as several studies were abortive by using OEC transplantation therapy for central nervous system (CNS) injuries $[12,13]$. These studies revealed that the inability of damaged neurons to regrow is likely to be attributed to transplanted OECs that either did not support or failed to guide neural regeneration [12]. With advances in regenerative medicine and neurobiology, this view is facing a great challenge. It is well known that in CNS injuries or neurodegenerative disorders, numerous neural cells hardly elude apoptotic fate to distinct damages. Consequently, this results in formation of an extrinsic inhibitory environment within CNS lesions, in which various growth inhibitory factors, for example interleukin- $1 \beta$, chemokine (C-X-C motif) ligand (CXCL)8, Nogo, oligodendrocyte-myelin glycoprotein, reactive oxygen species, and repulsive guidance molecule $\alpha$ are present and seriously inhibit neuronal survival and axonal regrowth [14-21]. Of note, the majority of these harmful molecules directly originate from degenerating or dying neurons and apoptotic neural debris $[8,9,22]$. Thus, rapid and efficient clearance of these deteriorated cell products is essential in creating a microenvironment beneficial for neuron survival and axonal regeneration following CNS damages or degeneration. More importantly, efficient removal of these degenerated tissues/cells also avoids the diffusion of damaging degradation products into tissue surroundings within CNS lesions, which could further exacerbate nerve damage or axonal degeneration [22]. Therefore, establishing an ideal, rapid, and effective strategy for the removal of degenerated cell debris for neuron survival, axonal regeneration, and subsequent neural function recovery is crucial. Based on our recent studies and some previous reports by other groups [8, 9, 23-26], increasing attention has been paid to OEC-mediated phagocytosis in CNS injury and degeneration, owing to their unique attributes. Thus, it is likely that OECs are potential candidates for therapeutic treatments.

Regardless of the phagocytic function of OECs in creating a microenvironment suitable for axonal regeneration, it is still unknown how to improve effectively OEC phagocytic activity without triggering undesirable consequences. Therefore, it is essential to develop an ideal, simple, and effective approach for the activation of OECs that contributes to the clearance of degenerated cell debris.

Lipopolysaccharide (LPS) is the major component of the outer membrane of Gram-negative bacteria; it also increases the negative charge of the cell membrane and helps stabilize the overall membrane structure $[27,28]$. Importantly, LPS elicits a strong response from the normal animal immune system and promotes the secretion of proinflammatory cytokines by binding the CD14/Toll-like receptor 4 (TLR4)/Myeloid differentiation factor 2 receptor complex in many cell types $[29,30]$. A growing body of evidence indicates that OECs can be activated by LPS, resulting in cellular stress response, release of cytokines, production of reactive oxygen species, and upregulation of phosphatidylserine receptor (PSR), which is involved in mediating the engulfment of apoptotic cell debris $[9,31,32]$. Curcumin (CCM) has been used as a traditional medicine in far-eastern countries such as China and India for centuries [33]. Several research groups have shown antiinflammatory effects of CCM in gastrointestinal diseases and cognitive disorders, as well as beneficial outcomes in psychiatric and anticancer settings [34-39]. Moreover, CCM can also exert distinct beneficial effects in antiproliferation, antioxidation, and antiapoptosis in a wide variety of cells [40-42]. In addition to the reported benefits, CCM is also known to possess neuroprotective properties [43, 44]. Strikingly, the biological effects of CCM are mainly dosage dependent and cell-type specific [45-47]. For instance, at high concentrations $(>40 \mu \mathrm{M}), \mathrm{CCM}$ induces cell apoptosis in diverse cancer cells [48], whereas low doses of CCM (0.1$0.5 \mu \mathrm{M})$ can stimulate cell proliferation in neural progenitor cells [46]. Within the injured spinal cord, CCM has been shown to exert anti-inflammatory and antioxidative effects at concentrations of $40-60 \mathrm{mg} / \mathrm{kg}$ [49]. Excitingly, several recent reports have demonstrated that CCM can improve OEC proliferation, migration, morphologic changes, and phagocytic activity [45]. In spite of growing interest in the investigation of the activation characteristics of OECs, particularly in the engulfment of apoptotic or degenerated cell debris, caused by $\mathrm{CCM}$ or other molecules, the strategies that further potentiate the beneficial behavior of OECs in the rapid clearance of degenerated cell debris is still unknown. However, whether activated OECs with phagocytic capacity are able to promote the growth of neurons in the CNS is unclear. More importantly, mechanisms underlying the cellular events are not entirely understood.

In the current study, we present an efficient induction approach, together with molecular and cellular evidence demonstrating that co-stimulation of OECs with LPS and CCM potentiates the phagocytic activity of OECs by effectively triggering their activation. Three significant breakthroughs were observed. First, the combination of the 2 molecules can effectively induce the activation of OECs without diminishing 1) its beneficial attributes, and 2) elevation of undesirable effects. Second, the activation of OECs by the combination of LPS and $\mathrm{CCM}$ can efficiently promote neuronal growth under hostile conditions. Third, transglutaminase-2 (TG2) and PSR may be involved in the cellular event. Importantly, OECs activated 
by LPS and CCM to clear degraded neural debris may open new avenues towards a potential therapy to repair an injured $\mathrm{CNS}$ or in the treatment of neurodegenerative diseases.

\section{Materials and Methods}

\section{Main Reagents}

Dulbecco's modified Eagle's medium (DMEM)/F12, fetal bovine serum (FBS), trypsin, DMEM, G5 supplement, B27 supplement, Neurabasal medium, Hanks' balanced salt solution (HBSS), collagenase, and normal goat serum were purchased from Gibco (Carlsbad, CA, USA); LPS, BrdU, CCM, forskolin, penicillin $\mathrm{G}$, streptomycin, glutamine, poly-Llysine (PLL), 3-(4,5)-dimethylthiazol-2-yl)-2,5dihenyltetrazolium bromide (MTT), ethylenediaminetetraacetic acid, HEPES, cystamine (Cyst), and bovine serum albumin were purchased from Sigma-Aldrich (St. Louis, MO, USA); neutrophil elastase (NE) was from R\&D (Minneapolis, MN, USA); anti- $\gamma$-tubulin and anti-Tuj-1 antibody, antiglial fibrillary acidic protein (GFAP) antibody, anti-S100 antibody, and anti-p75 antibody were purchased from Abcam (Cambridge, MA, USA); anti-CXCL1, anti-CXCL2, antitumor necrosis factor (TNF)- $\alpha$, and anti-TLR4 antibody were purchased from Santa Cruz (Santa Cruz, CA, USA); TG2 and PSR antibodies were from Cell Signaling (Danvers, MA, USA). The RNA extract kit was purchased from Takara (Shiga, Japan); Revert Aid First strand cDNA synthesis kit was purchased from Thermo Scientific (Rockford, IL, USA); QPCR kit was purchased from Invitrogen (Shanghai, China); 4',6-diamidino-2phenylindole and a bicinchoninic acid kit was from Qiagen (Hilden, Germany); a chemiluminescence Western blotting kit was purchased from Plus (Roche, Mannheim, Germany); Fluor488-conjugated goat anti-mouse IgG, and Fluor594conjugated donkey antirabbit were purchased from Molecular Probes (Eugene, OR, USA). Thirty-fivemillimeter dishes, cell culture plates, plastic coverslips, and flasks were all purchased from Corning (Corning, NY, USA).

\section{Primary OEC and Neuron Culture}

All procedures conducted on animals were approved by the Animal Experimentation Ethics Committee of Xi'an Jiaotong University and were consistent with the China Code of Practice for the Care and Use of Animals for Scientific Purposes. Primary OECs were prepared from mouse olfactory bulbs at 2.5 months of age and further purified as described previously [50], with minor modifications. Briefly, the outer nerve fiber and granular layers of olfactory bulbs were dissected from male mice and rinsed twice in HBSS without $\mathrm{Ca}^{2+}$ $\mathrm{Mg}^{2+}$. Then, tissues were minced using an iris scissor and digested using a solution composed of $0.25 \%$ trypsin and
$0.2 \%$ dispase II for $25 \mathrm{~min}$ at $37{ }^{\circ} \mathrm{C}$. The digested tissue was triturated, and filtered through an $80-\mu \mathrm{m}$ metal mesh. The cell suspension was resuspended in DMEM/F12 with $10 \%$ FBS and plated on PLL-coated flasks and cultured at $37{ }^{\circ} \mathrm{C}$ in a humidified incubator at $5 \% \mathrm{CO}_{2}$. Half the media were changed every 3 days. When confluence of OECs reached $85 \%$, cell purification was conducted by differential cell adhesiveness. The purified cell suspension was collected and reseeded onto $25-\mathrm{cm}^{2}$ PLL-coated culture flasks and incubated with $10 \%$ FBS-containing DMEM/F12 supplemented with $2 \mu \mathrm{M}$ forskolin for 2 days. Subsequently, cells were maintained in DMEM/F12 media containing $1 \%$ G5 supplement for further experiments.

As for neuron cultures, primary cultures of spinal cord neurons were prepared from embryonic 12- to 14-day-old red fluorescent protein (RFP) transgenic or normal mice pursuant to the procedure as previously described by Yang et al. [8, 51]. Briefly, the spinal cords were dissected and washed with ice-cold HBSS without $\mathrm{Ca}^{2+}$ and $\mathrm{Mg}^{2+}$. Subsequently, tissues were trypsinized with $2 \mathrm{~mL} 0.125 \%$ (w/v) trypsin at $37^{\circ} \mathrm{C}$ for 25 min before mechanical trituration. The dissociated cells were then collected by centrifugation and diluted to an approximate initial plating density of $2 \times 10^{5}$ cells $/ \mathrm{cm}^{2}$ with Neurabasal medium supplemented with $2 \%$ B27, and plated into either 35-mm Petri dishes or coverslips coated with PLL. Cultures were maintained in an incubator at $37{ }^{\circ} \mathrm{C}$ in a humidified atmosphere of $5 \% \mathrm{CO}_{2}$. The cultures were maintained and prepared for further experiments.

\section{Treatment of OECs}

To examine if the combination of LPS and CCM contributes to the beneficial activation of OECs, OECs were reseeded on coverslips, 35-mm dishes, and 96-well plates, and subsequently divided into the following groups: 1) normal OECs; 2) OECs treated with $1 \mu \mathrm{g} / \mathrm{ml}$ LPS; 3) OECs treated with $1 \mu \mathrm{M}$ curcumin; 4) OECs treated with $1 \mu \mathrm{g} / \mathrm{ml}$ LPS and $1 \mu \mathrm{M}$ curcumin; 4) OECs pretreated with Cyst (an inhibitor of TG2) and NE (a cleavage enzyme of PSR) prior to constant co-stimulation with $1 \mu \mathrm{g} / \mathrm{ml}$ LPS and $1 \mu \mathrm{M}$ curcumin. After 1 , 2 , and 3 days, cells were processed for the following experiments.

\section{Preparation of Degenerative Neuronal Debris}

Neural debris was prepared from RFP mice. Apoptotic neuronal bodies and axon debris was harvested by scratching with a cell blade and further trituration, according to our previously described methods [8], resulting in fragmented debris consisting of microtubules and neurofilaments. Degenerative neuronal debris was stored at $-80{ }^{\circ} \mathrm{C}$ until required for use. 


\section{MTT Assay}

For determination of OEC activation, MTT assay was used to assess the proliferative capacity of OECs. In brief, OECs were plated in 96-well plates and treated for 1, 2, and 3 days. MTT, $5 \mathrm{mg} / \mathrm{ml}$, was added directly to cultures. After $4 \mathrm{~h}$ incubation at $37^{\circ} \mathrm{C}$, the medium from each well was gently removed by aspiration and $200 \mu \mathrm{l}$ dimethylsulfoxide was added to each well followed by incubation and shaking for $10 \mathrm{~s}$, to dissolve the insoluble formazan. Subsequently, OD values were measured in a microplate reader at $490 \mathrm{~nm}$ to determine the number of viable cells at indicated time points. All data presented herein were obtained from 3 independent experiments.

\section{BrdU Incorporation Assay}

The increase in the proliferative ability of OECs is an important feature of their activation. To assess the proliferation of OECs treated under different conditions, BrdU $(5 \mu \mathrm{M})$ was added to the culture medium for $18 \mathrm{~h}$ and cells were cultured for 1, 2, and 3 days. After removal of the supernatant, cells were fixed with $4 \%$ paraformaldehyde, and treated with $2 \mathrm{~N}$ hydrochloric acid for $30 \mathrm{~min}$ at $37^{\circ} \mathrm{C}$ to denature DNA for further immunostaining. Subsequently, immunostaining procedures were performed as previously described [52].

\section{Real-Time Polymerase Chain Reaction}

Total RNA was extracted from OECs at 2 different treatment time points (days 1 and 3) using RNAeasy (Qiagen), according to manufacturer's instructions. Glyceraldehyde 3phosphate dehydrogenase was used as the internal control and levels of target mRNAs were normalized against glyceraldehyde 3-phosphate dehydrogenase mRNA. Two micrograms of each total RNA were reverse transcribed into cDNA using PrimeScript RT reagent kit (Takara). The polymerase chain reaction (PCR) reaction conditions were as follows: $94{ }^{\circ} \mathrm{C}$ for $5 \mathrm{~min}, 40$ cycles at $94{ }^{\circ} \mathrm{C}$ for $30 \mathrm{~s}, 58^{\circ} \mathrm{C}$ for $30 \mathrm{~s}$, and $72{ }^{\circ} \mathrm{C}$ for $1 \mathrm{~min}$. PCR was performed in triplicate and repeated 3 times. Genes of interest were $\mathrm{Cxcl1}$, Cxcl2, TLR4, tnfa, tg2, and psr. The mRNA levels were quantified by SYBR green-based quantitative real-time PCR (Takara) using an ABI Prism 7900 HT (Applied Biosystems, Foster City, CA, USA). Results were confirmed in at least 3 separate analyses. Primers sequences are listed in Table 1.

\section{Western Blots}

Differently treated OECs were lysed in RIPA buffer for $30 \mathrm{~min}$ on ice and crude extracts were sonicated to shear DNA. Protein samples were harvested by centrifugation at $12,000 \times g$ and clarified lysates were measured using a bicinchoninic acid protein assay. The supernatant was collected and Western blots
Table 1 Real-time polymerase chain reaction primers

\begin{tabular}{lll}
\hline Gene & Forward primer & Reverse primer \\
\hline$C X C L 1$ & agaacatccagagtttgaaggtga & gtggctatgacttcggtttgg \\
$C X C L 2$ & tggttcagaggatcgtccaaa & caggagcccatgttcttcctt \\
$T N F \alpha$ & gacgtggaagtggcagaagag & tgccacaagcaggaatgaga \\
$T L R 4$ & atcatccaggaaggcttcca & gctgcctcagcaaggacttct \\
$T G 2$ & tatggccagtgctgggtccttcgcc & ggctccagggttaggttgagcagg \\
PSR & gactctggagcgcctaaaaa & ccctgaactaaggcattcca \\
GAPDH & tgaggccggtgctgagtatgtcg & ccacagtcttctgggtggcagtg \\
\hline
\end{tabular}

performed according to the protocol as described previously [51]. The following antibodies were used: CXCL1, CXCL2, p75, TLR4, TNF- $\alpha$, TG2, and PSR. $\beta$-Actin was used as an internal control. After washes in phosphate-buffered saline (PBS), immunoblots were visualized using enhanced chemiluminescence. Densitometric analysis of bands was repeated 4 times and integrated densitometry value (IDV) was calculated.

\section{Flow Cytometry}

Cell-cycle evaluation was determined by flow cytometry (ProfileII, Coulter, Brea, CA, USA) according to a previously described protocol $[52,53]$. Briefly, after OECs were treated under different conditions for the 3 abovementioned time points, cells were trypsinized with $0.125 \%$ trypsin and $0.02 \%$ ethylenediaminetetraacetic acid, collected, and washed twice in ice-cold PBS. Single-cell suspension was achieved by gentle trituration up and down, and filtration through an 80- $\mu$ m nylon mesh before analysis. Cells were then transferred to 1.5-ml Eppendorf tubes and fixed with $70 \%$ ethanol at $4{ }^{\circ} \mathrm{C}$ overnight. Subsequently, cells were incubated with $10 \mu \mathrm{l}$ RNase A $(5 \mathrm{mg} / \mathrm{ml})$ at $37{ }^{\circ} \mathrm{C}$ for $30 \mathrm{~min}$, and cellular DNA was stained with propidium iodide (100 mg/ml) for $30 \mathrm{~min}$. Cells were washed in PFN (PBS buffer supplemented with $10 \%$ FCS and $0.02 \%$ sodium azide) 3 times and resuspended in PFN buffer prior to flow cytometry analysis. The proliferation index (PI) was calculated using the following equation: $\mathrm{PI}(\%)=(\mathrm{S}+\mathrm{G} 2 / \mathrm{M}) /(\mathrm{G} 0 / \mathrm{G} 1$ $+\mathrm{S}+\mathrm{G} 2 / \mathrm{M}) 100 \%$.

\section{Phagocytosis Assays}

To determine if LPS and CCM synergistically strengthen OEC phagocytic capacity, OECs were seeded in $35-\mathrm{mm}$ culture dishes prior to pretreatment with LPS at $1 \mu \mathrm{g} / \mathrm{ml}$ and $1 \mu \mathrm{M}$ CCM for $12 \mathrm{~h}$, and incubated with the abovementioned degeneration debris for 1,3 , and 5 days. In parallel, pretreatment of OECs with $100 \mu \mathrm{M}$ Cyst and $100 \mathrm{ng} / \mathrm{ml} \mathrm{NE}$ prior to exposure to LPS and CCM was conducted to further substantiate the hypothesis. The volume of cell degeneration debris administrated into OEC cultures was measured, and the ability of 
OECs to engulf the degenerated cell debris was assessed by a phagocytic index, according to our previously described protocol [8]. The results were obtained from 3 independent experiments.

\section{Distinct Treatment of Neurons}

To determine if OECs treated with the combination of LPS and CCM actively contribute to neuron growth under degenerated neuron debris culture conditions, neurons cultured on coverslips were divided as follows: 1) normal neurons co-cultured with OECs; 2) normal neurons co-cultured with OECs supplemented with degenerative neuronal debris; 3) normal neurons co-cultured with OECs exposed to LPS and CCM supplemented with degenerative neuronal debris; 4) normal neurons co-cultured with OECs exposed to LPS supplemented with degenerative neuronal debris; 5) normal neurons co-cultured with OECs exposed to CCM-supplemented degenerative neural debris; 6) normal neurons treated with LPS; 7) normal neurons treated with CCM; 8) normal neurons pretreated with Cyst and NE prior to exposure to LPS and CCM; 9) normal neurons treated with LPS and CCM in the presence or absence of degenerated neural debris; 10) normal neurons treated with degenerative neuronal debris alone. Cells from all groups were maintained at $37^{\circ} \mathrm{C}$ in a humidified $5 \%$ $\mathrm{CO}_{2}$ atmosphere for 3 days. Notably, OECs were pretreated with the LPS + CCM, LPS alone, or CCM alone for $12 \mathrm{~h}$ prior to co-culture with neurons. Concomitantly, cells were processed for following different examinations.

\section{Cell Count}

To determine the survival of neurons under the different treatments mentioned in the previous section, all Tuj-1-positive cells, regardless of their phenotypes, were counted as described previously [8]. Cell count was performed by a person blinded to the experimental setting. From each coverslip 15 randomly chosen fields were counted. In all analyses, data represent the mean \pm SEM of 4 independent experiments. Each performed experiment was comprised of 4 coverslips and the obtained result considered as the neuron survival index.

\section{Immunofluorescence}

All cells of all groups and treatments were fixed with $4 \%$ paraformaldehyde for 15-20 min, treated with $0.01 \%$ Triton X-100 (for verification of OEC identity, not treated with Triton X-100), and blocked with $3 \%$ normal donkey serum in $0.01 \mathrm{M} \mathrm{PBS}$ at room temperature (RT) for $30 \mathrm{~min}$. The following primary antibodies were used: rabbit anti-Tuj-1 (1:400); mouse anti-p75 (1:200); goat anti-GFAP (1:500); mouse anti-S100 (1:300); mouse anti-OX42 (1:200); rabbit
anti-TG2 (1:400); rabbit anti-PSR (1:200). Incubation of primary antibodies was carried out at $4{ }^{\circ} \mathrm{C}$ overnight. After washing 3 times in PBS, cells were incubated with corresponding fluorescence-conjugated secondary antibodies (Alexa Fluor 488 donkey antirabbit IgG antibody for Tuj-1, TG2 and PSR, 1:400 dilution; Alexa Fluor 488-conjugated goat antimouse IgG for p75, 1:400 dilution; Alexa Fluor 594 donkey antigoat IgG antibody for GFAP, 1:800 dilution; Alexa Fluor 594 donkey antimouse IgG antibody for S100 and OX42, 1:500) for $1 \mathrm{~h}$ at RT. Thereafter, counter staining using 4,6-diamidino-2-phenylindole $(2 \mu \mathrm{g} / \mathrm{ml})$ at RT for $15 \mathrm{~min}$ was performed and fluorescence evaluated using a confocal epifluorescence microscope (Leica, Wetzlar, Germany). Immunofluorescence was performed in triplicate and representative images were captured.

\section{Statistics}

All data are expressed as mean $\pm \mathrm{SD}$ or mean $\pm \mathrm{SEM}$. Statistical significance between groups was determined by 1way analysis of variance, followed by Scheffe's post hoc analysis. A $p$-value $<0.05$ was considered statistically significant.

\section{Results}

\section{Characterization and Identification of OECs and Neurons}

To investigate whether synergistic LPS and CCM signaling potentiates activation of OECs, we first cultured, characterized, and identified primary mouse OECs and spinal cord neurons. The morphology of OECs at 5 days and 7 days after purification was evaluated by phase-contrast microscopy (Fig. 1a, b). Purified OECs were subsequently stained by immunofluorescence and were identified as positive for $\mathrm{p} 75$, GFAP, and S100 (Fig. 1c-f), characteristic markers of OECs. Of note, these cells did not express OX42, a specific marker for microglial cells (Fig. 1g), suggesting that purified OECs are not contaminated with microglia and that the isolation procedure results in high purity. For primary neurons, we found that after 7 days of culture these cells were of irregular shape, showed very thin and long extensions, and eventually formed a more intricate network (Fig. 1h). In addition, immunofluorescence staining demonstrated that the cells were, indeed, neurons (Fig. 1i), and thus were used subsequently for preparation of degenerated neuron debris and further experiments.

\section{Enhancement of OEC Activation Through Co-stimulation by LPS and CCM}

To test whether LPS and CCM can synergistically enhance OEC activation, we selected 4 characteristic molecules 
Fig. 1 Morphological and biochemical characteristics of primary olfactory ensheathing cells (OECs) and neurons. (a, b, h) Phase-contrast microscopy showing morphology of primary OECs after purification at 5 and 7 days of culture, as well as spinal cord neurons at 7 days. (c, $\mathbf{d}, \mathbf{e}, \mathbf{g}$ ) Immunofluorescence revealed expression of $\mathrm{p} 75$, glial fibrillary acidic protein (GFAP), and $\mathrm{S} 100$, but not OX-42, in purified OECs. h More importantly, cells coexpressed p75 and GFAP. i In addition, immunofluorescence demonstrated that all Tuj-1positive cells are neurons. Scale bars $=100 \mu \mathrm{m}$
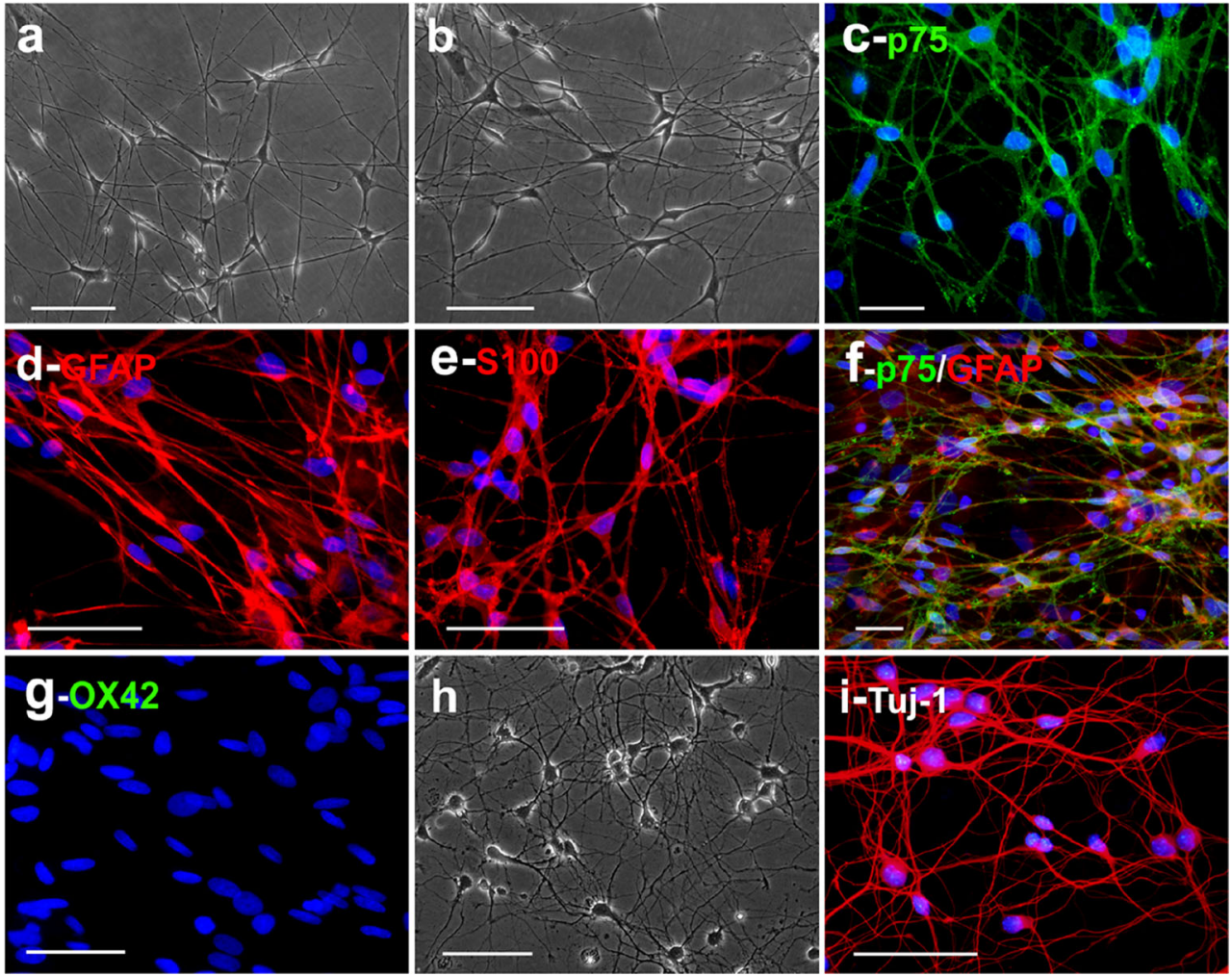

(CXCL1, CXCL2, TNF- $\alpha$, and TLR4) crucial for OEC activation and compared their expression profiles in OECs after each treatment. As shown in Fig. 2a-d, after 1 and 3 days of treatment with LPS alone, CCM alone, or LPS + CCM, mRNA levels of selected markers were drastically upregulated in OECs, although no significant difference was found in case of CXCL2 and TLR4 expression after 1 day of treatment. In contrast, the combination of LPS + CCM significantly upregulated the aforementioned molecules, displaying an approximately 4- to 500-fold increase compared with the control group or the LPS alone and CCM alone groups. Consistently, Western blot revealed a significant increase in the expression of these molecules in OECs. Strikingly, expression of the selected molecules showed a time-dependent increase, that is, they dramatically increased within 3 days when compared with cells treated with LPS or CCM alone (Fig. 2e, f). Overall, these data suggest that LPS and CCM can promote OEC activation.

\section{LPS and CCM Synergistically Potentiate the Proliferation of OECs}

In addition to the characteristic marker expression associated with cell activation, elevated proliferation is also one of the crucial hallmarks of activated OECs. Thus, cell proliferation was assessed and quantitated using $\mathrm{BrdU}$ incorporation assays. After 3 days of treatment, the number of $\mathrm{BrdU}^{+} \mathrm{OECs}$ in the LPS + CCM group was significantly increased compared with LPS alone, CCM alone, or the control group (Fig. 3a). Also, quantification analysis revealed that there was a significant difference in the percentage of $\mathrm{BrdU}^{+} \mathrm{OECs}$ among the 3 treatment groups $(18.1 \% \pm 2.8 \%$ for control; $25.0 \% \pm 3.8 \%$ for CMM alone; $22.5 \% \pm 1.8 \%$ for LPS alone; and $38.2 \% \pm 5.2 \%$ for LPS + CCM) (Fig. 3b) ( $p<0.01$ and $p<0.001 v s$ the corresponding controls), suggesting that LPS and CCM co-stimulation enhances OEC activation (Fig. 3b). To further substantiate this claim, an MTT assay was also used to evaluate cell proliferation. Consistently, LPS and CCM significantly enhanced OEC proliferation compared with the LPS alone and CCM alone groups, and the untreated control group, although both LPS and CCM alone slightly elevated OEC proliferation (Fig. 3c). Furthermore, cell-cycle distribution was determined by flow cytometric analysis of propidium iodide-stained cells. Flow cytometry data showed the representative cell cycle changes of OECs with the 3-day treatment (Fig. 3d). The changes in cell cycle for OECs after the indicative treatments of 3 days are shown in Fig. 3d. Notably, the percentage of OECs in the G2/M phase was significantly enhanced when treated with the combination of CCM and LPS compared with LPS alone, CCM alone, and control $(p<0.01, p<0.001)$. In contrast, cell populations in the G1 phase were clearly reduced in OECs. Collectively, these data suggest that the combination of LPS and $\mathrm{CCM}$ can efficiently promote the transition from $\mathrm{G} 1$ to $\mathrm{S}$ phase and G2/M phase, which represents cell proliferative ability. 
Fig. 2 Cooperative effects of lipopolysaccharide (LPS) and curcumin $(\mathrm{CCM})$ on the activation of olfactory ensheathing cells (OECs). a-d Real-time polymerase chain reaction showed that mRNA levels of chemokine (C-X-C motif) ligand (CXCL)1, CXCL2, tumor necrosis factor (TNF)- $\alpha$, and Toll-like receptor (TLR)4 increase in OECs stimulated with LPS alone, CCM alone, or the combination of both for 1 and 3 days, respectively. Notably, the combination of LPS and CCM significantly increased the expression levels of these molecules in OECs. All data are reported as means \pm SEM. $* p<0.05$ compared with the relevant controls; $* * p<0.01$; and *** $p<0.001$. e Western blots further support expression of the 4 characteristic molecules in OECs under the indicated conditions. $\mathbf{f}$ $\beta$-Actin served as a loading control of total proteins.

Strikingly, the expression levels of the 4 molecules in OECs stimulated with the combination of LPS and CCM showed a timedependent upregulation
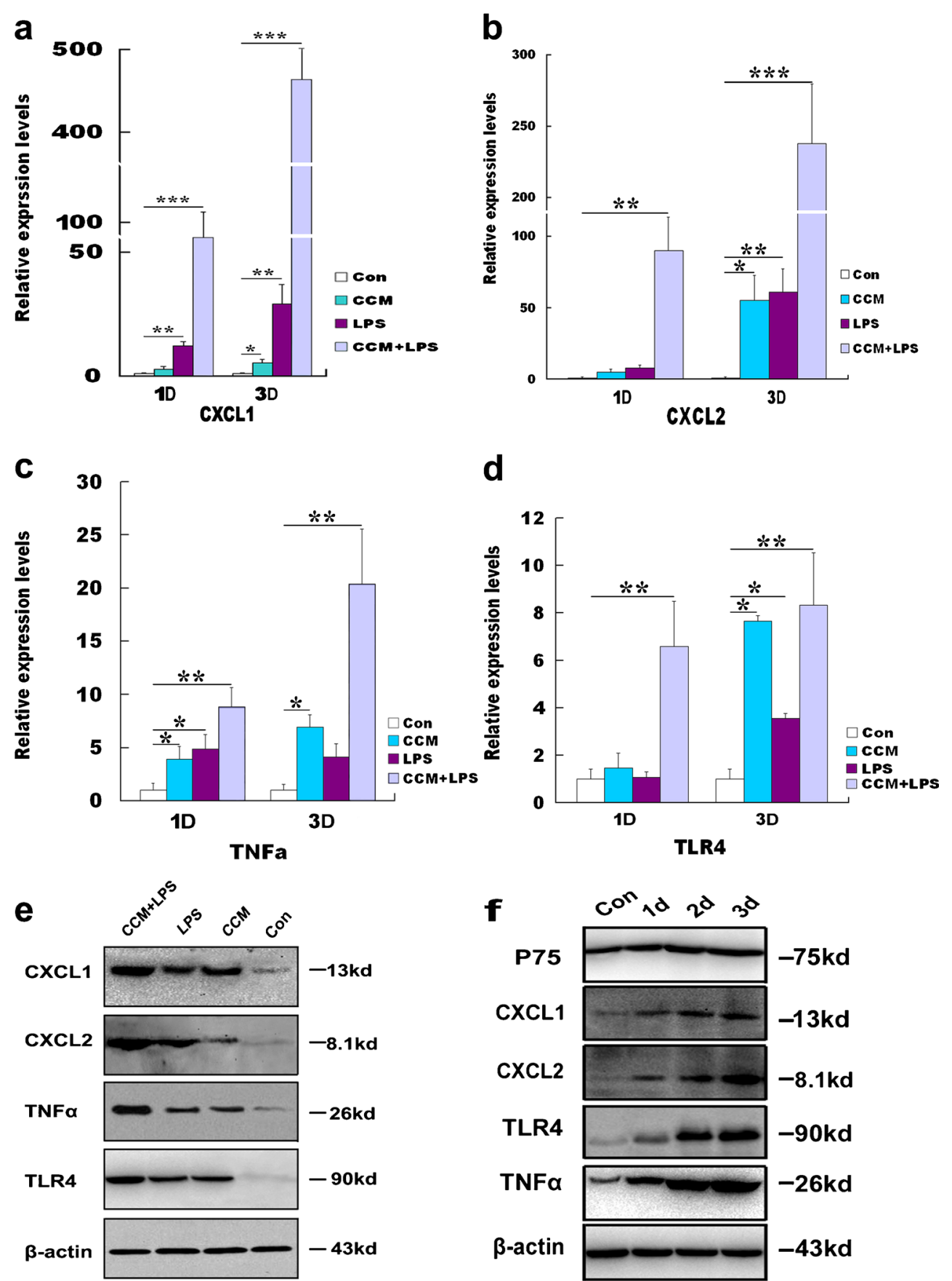

\section{The Effects of LPS and CCM on the Phagocytic Ability of OECs Targeting Degenerated Neural Debris}

To validate whether the combination of LPS and CCM effectively strengthens activation of OECs, resulting in cellular phagocytosis, we investigated ingestion of degenerated RFP neural debris by OECs pretreated under the aforementioned conditions in vitro. Phase-contrast microscopy revealed that the degenerated neural debris was present inside the OEC cytoplasm. In comparison, the amount of red cell debris within OECs treated with LPS and CCM was much more than within those treated under other conditions (Fig. 4a). To verify the uptake of cell debris, the images taken by a differential interference contrast microscopy were analyzed. Consistently, the most abundant punctuate or granular debris was present in the cytoplasm of OECs pretreated with the combination of LPS + CCM. However, some debris was also present in the cell cytoplasm of the OECs in other treatment groups. The engulfed debris showed punctuate attributes (Fig. 4b). To confirm that LPS and CCM can enhance OEC activation, which consequently results in an increase in OEC phagocytosis, the phagocytic capacity of OECs was further assayed according to 

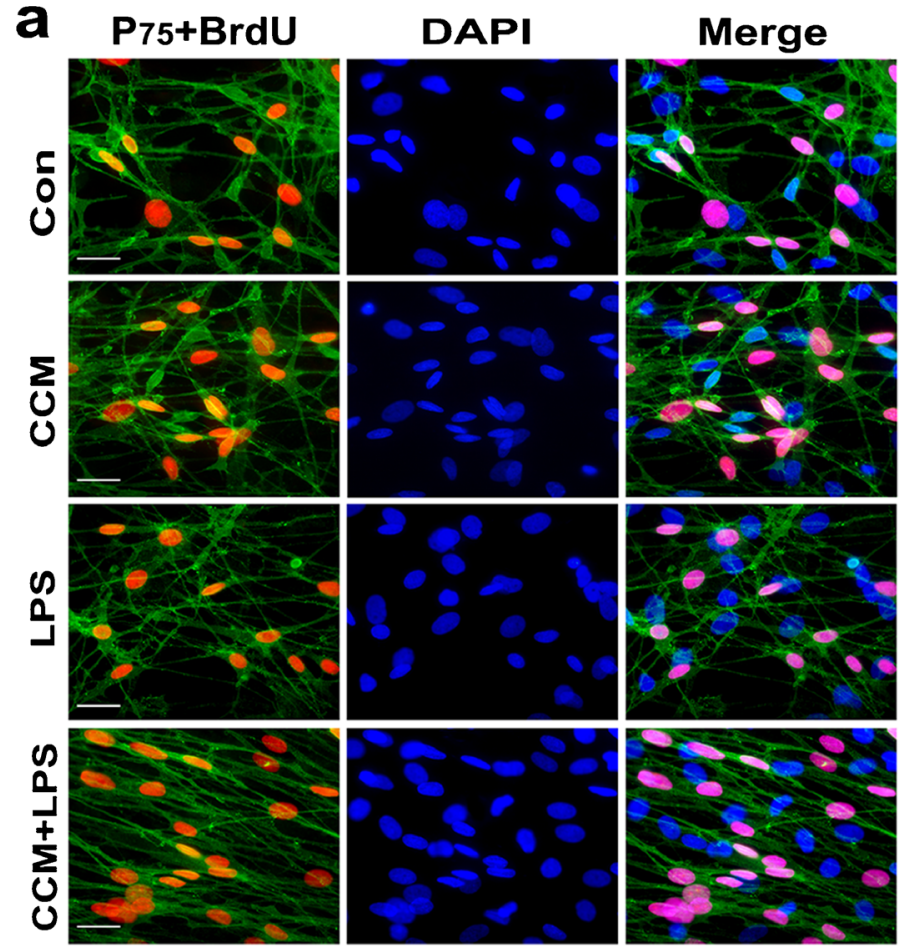
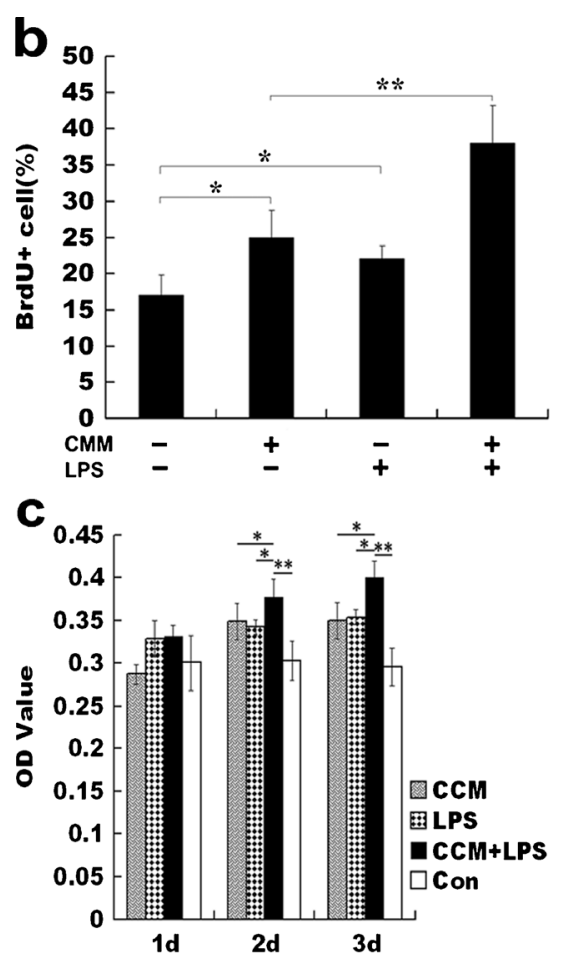

d

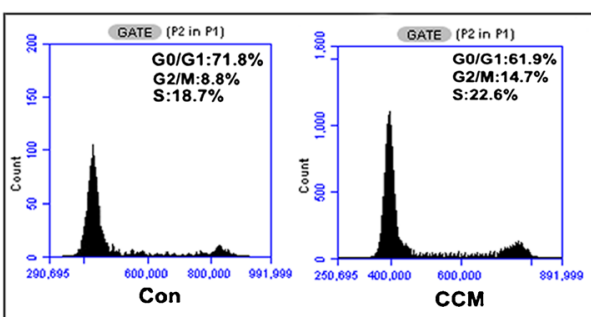

Fig. 3 Co-stimulatory effects of lipopolysaccharide (LPS) and curcumin $(\mathrm{CCM})$ on olfactory ensheathing cell (OEC) proliferation. a Photomicrographs of BrdU incorporation into p75-positive cells, to assess OEC proliferation after different treatments, as indicated. b Quantification analysis of BrdU-positive OECs with the indicated treatments. All data are reported means $\pm \mathrm{SEM}$, and representative of 3 independent experiments with similar findings. ${ }^{*} p<0.05$ and $* * p<0.01$ $v s$ controls (Con). c Quantification of the proliferation rate in 3-(4,5)dimethylthiazol-2-yl)-2,5-dihenyltetrazolium bromide assays revealed that the combination of LPS and CCM significantly promoted OEC proliferation compared with other treatment conditions. Enhancement of cell proliferation was time-dependent. d OECs were treated under the indicated conditions for 3 days and analyzed by flow cytometry. G0/ G1, G2/M, and S represent cell phase, and the corresponding data are shown in each panel. Statistical analysis of the percentage of OECs in G2/ $M$ phase shows significant difference when treated with the combination of LPS + CCM compared with other treatments (lower bottom panel, right). Each phase was calculated by using the cell ModiFIT program. All data are reported as means \pm SEM. $* p<0.05$, $* * p<0.01$, and $* * * p<0.001 v s$ its relevant controls. DAPI $=4,6$-diamidino-2phenylindole. Scale bars $=20 \mu \mathrm{m}$

demonstrated that LPS and CCM can efficiently strengthen OEC phagocytosis of degenerated neuron debris.

\section{The Effects of OEC Activation by LPS and CCM on Neuron Growth}

We next examined whether cell debris removal by LPS- and CCM-treated OECs plays a role in promoting neuron growth. Spinal cord neurons were cultured for up to 3 days after treatment with degenerated neuron debris, and immunofluorescence was performed using Tuj-1 antiboy. As shown in and $p<0.01$, respectively; Fig. 4e). Thus, these results 

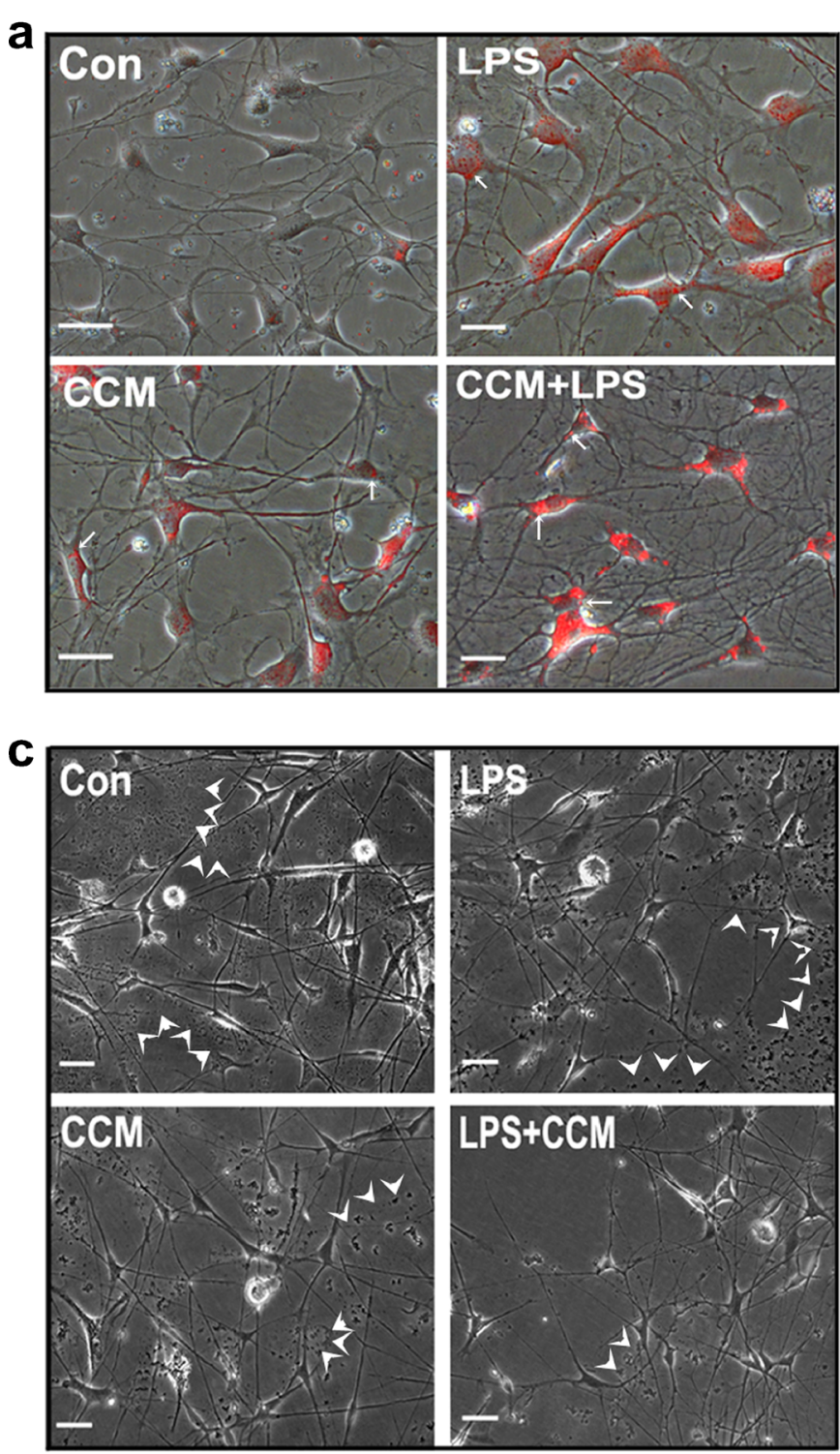

Fig. 4 The effect of lipopolysaccharide (LPS) and curcumin (CCM) on the phagocytic ability of olfactory ensheathing cells (OECs). a Phasecontrast photographs of engulfed degenerated neural debris by OECs treated with different conditions as indicated (red signals represent neural debris generated from red fluorescent protein mouse). b Differential interference contrast microscopy of engulfed degenerated neural debris by OECs treated with indicated conditions. c Representative photographs of phagocytic neural debris uptake by

Fig. 5a, almost all neurons exhibited poor morphology, with short neurites and little arborizations. Concomitantly, an abundant scattering of degenerated neuron debris resulting from dying, cultured neuronal cells appeared. In contrast, no apparent debris was observed around neurons under normal culture conditions. Notably, neurons with extended considerable and abundant branching and arborizations in neurites were present. When switched to unstimulated OEC co-culture, surviving neurons exhibited relatively better growth, even though degenerated cell debris was administered. Comparatively, it
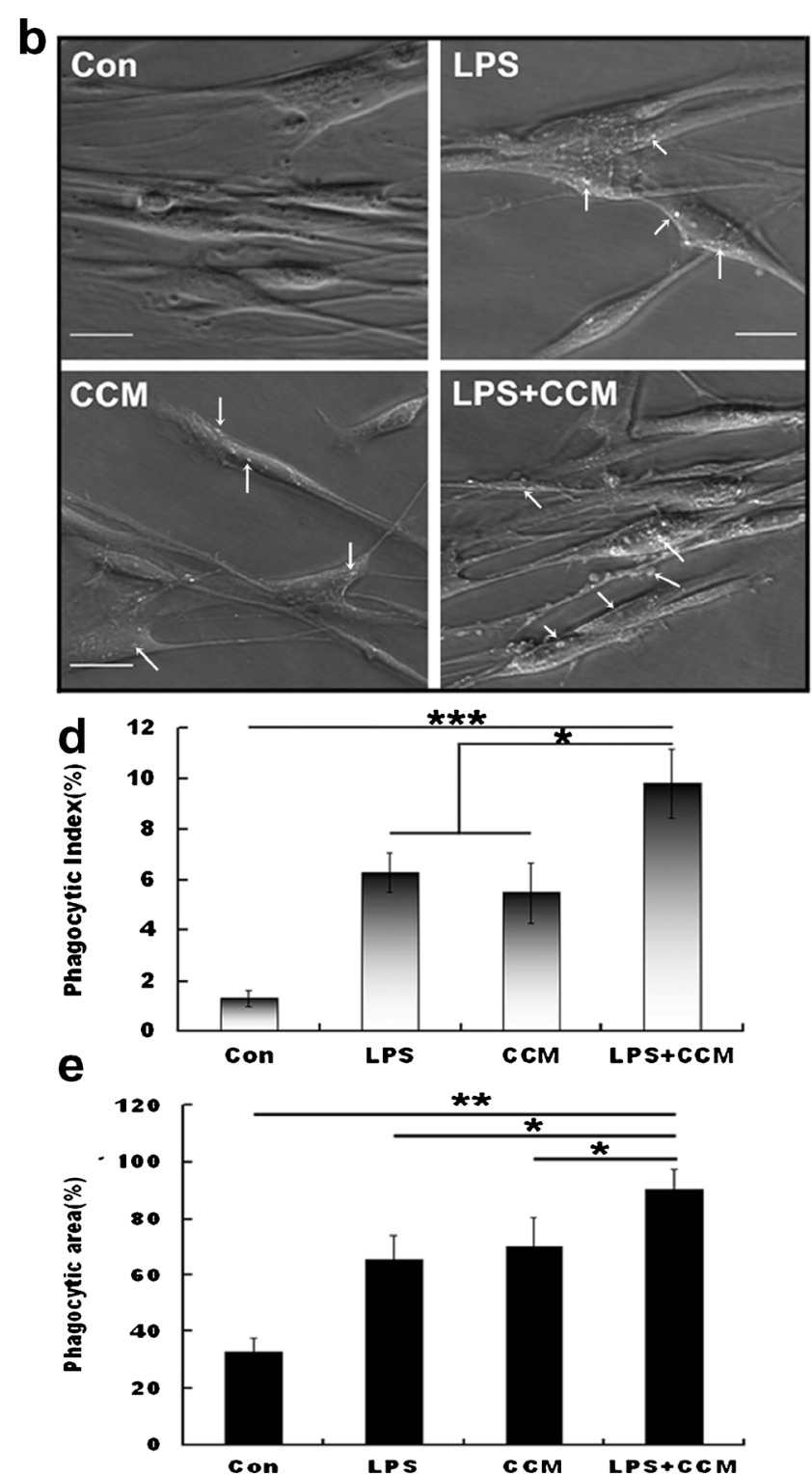

OECs in cultures under the indicated treatments. d Quantitative analysis of the relative number of engulfed debris by OECs under the indicated treatments. e Quantitative analysis of phagocytic areas in OEC cultures. $* * p<0.05 ; * * p<0.01$, and $* * * p<0.001$ compared with its corresponding controls (Con). Of note, arrows indicate ingested neural debris in cells. Arrowheads represent a progressive enlarged phagocytic area. Scale bars $=50 \mu \mathrm{m}$

seemed that more neurons and richer neurites were observable in absence of cell debris. With the addition of LPS or CCM alone, we also found moderate Tuj-1-positive cells and less cell debris in cultures. Moreover, most Tuj-1-positive cells exhibited longer process extension and richer arborizations than the control group. Notably, both LPS alone and CCM alone showed neither harmful nor promotive effects on neuron survival and neurite outgrowth in the presence or absence of degenerated cell debris (data not shown). Strikingly, when neurons were co-cultured with OECs treated with the 
Fig. 5 Clearance of degenerated debris by olfactory ensheathing cells (OECs) treated under different conditions allows neuron survival and neurite outgrowth. a Representative images of primary spinal cord neuron at low density following different treatments as indicated. b The photographs of neurons (green, Tuj-1 staining) cocultured with OECs (red, p75 staining) treated with the combination of curcumin (CCM) and lipopolysaccharide (LPS) in the presence or absence of degenerated neural debris. $\mathbf{c}, \mathbf{d}$ Quantitative assessment of neuron survival and neurite length, respectively, under indicated treatments. $* * p<0.01$ and $* * * p<0.001 v s$ their respective control (Con), analysis of variance post hoc test. Scale bars $=200 \mu \mathrm{m}$
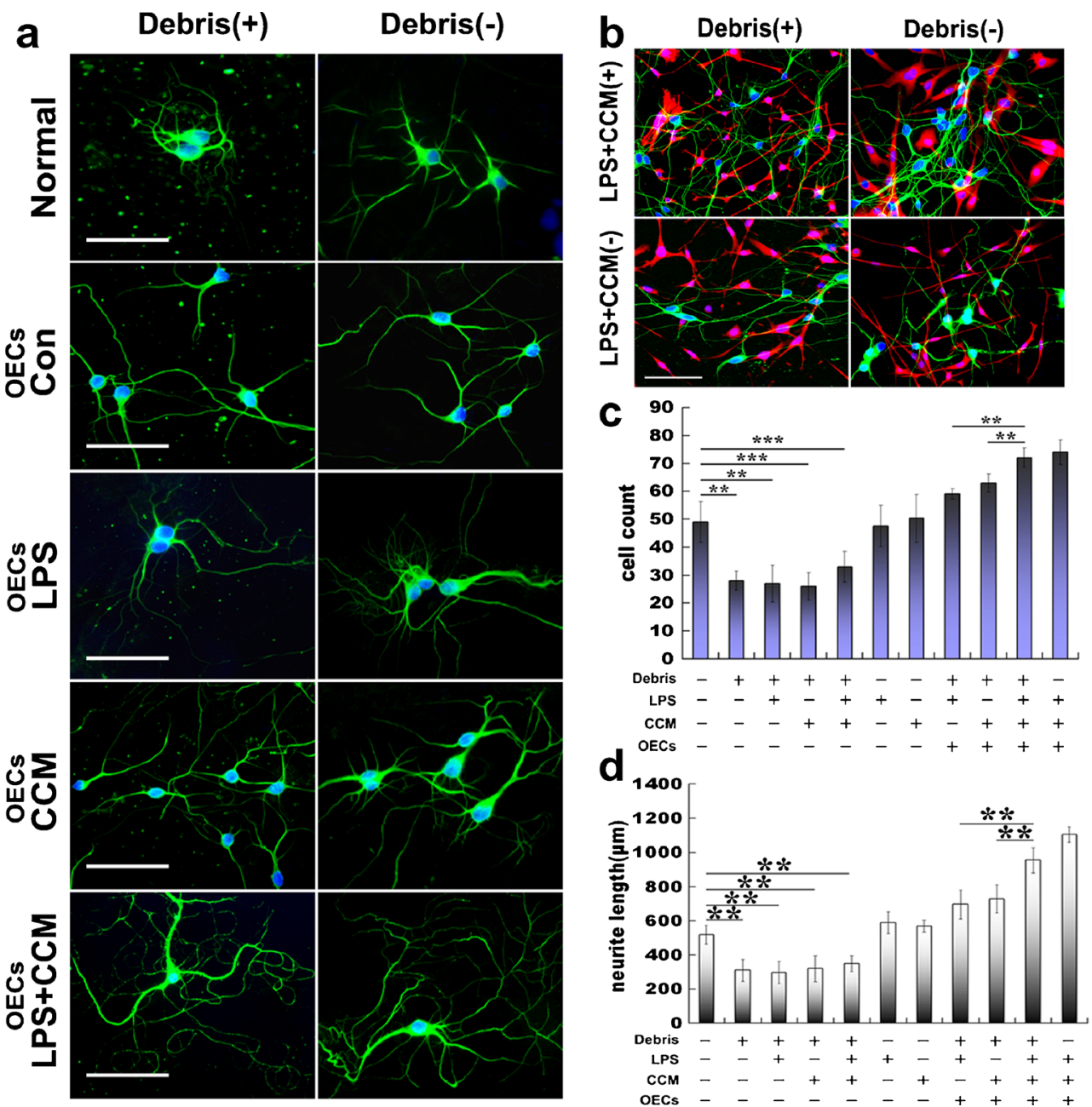

combination of LPS and CCM, almost all Tuj-1-positive cells exhibited longer and thicker processes, with an excessive degree of branching, and there were many more neurons in each visual field. Comparatively, no significant difference was detected between the 2 groups with or without the degenerated cells debris (lower panels in Fig. 5). Collectively, these data indicate that LPS and CCM synergistically enhance neuron survival and neurite outgrowth through enhancing activation of OECs.

To validate directly the response of neurons to OECs pretreated with the combination of CCM + LPS in the presence or absence of degenerated neural debris, we performed co-culture of neuronal cells with OECs, and determined the effects of the activated OECs on neuron outgrowth by means of Tuj-1 and p75 immunostaining. As shown in Fig. 5b, the combination of CCM + LPS remarkably promoted neuron (green, Tuj-1-positive) survival in the presence of degenerated neural debris, and almost all surviving cells were characteristically exuberant with pronounced longer neurite extensions compared with the corresponding control. Adversely, in the absence of CCM and LPS, a relative decrease in Tuj-1positive cells was found in the visual field, and these cells exhibited few processes with poor arborization. However, a small amount of scattered residual debris around the positive cells was seen. As for normal neurons co-cultured with OECs exposed to CCM and LPS, there were no observable differences in neurite extension except for the number of surviving neurons when compared with the group of degenerated neural debris. Strikingly, there was a significant difference in neuron survival and neurite outgrowth between normal neurons in the presence and absence of CCM and LPS.

Furthermore, enhancement of neuronal survival and neurite outgrowth by activated OECs (induced by the combination of LPS + CMM) was quantitatively analyzed by cell count and neurite assays. As shown in Fig. 5c, after culture for 3 days under the aforementioned conditions, the presence of degenerated cell debris suppressed neuron survival remarkably. This was evidenced by a significant decrease in living cells compared with the normal cultured neurons $(p<0.01)$. Unfortunately, the administration of LPS alone, CCM alone, or their combination (without OECs) did not remarkably alleviate the suppression from the degenerated cell debris. When co-cultured with OECs, neuron survival was significantly improved compared with the corresponding controls. Notably, 
the combination of LPS and CCM markedly enhanced neuron survival. Statistical analysis revealed that there was a significant difference when compared with the groups of LPS alone and CCM alone in the presence or absence of the degenerated neuron debris ( $p<0.01$ and $p<0.001$, respectively).

Besides neuronal survival, neurite length was also measured. As shown in Fig. 5d, average neurite lengths ( $\mu \mathrm{m})$ from the different groups were $520 \pm 55$ for the normal group; 310 \pm 64.3 for the debris group; $297 \pm 64.8$ for the debris + LPS group; $320.5 \pm 75.3$ for the debris + CCM group; $350 \pm 45.2$ for the debris + LPS/CCM group; $590 \pm 64.2$ for the debris + CCM/OEC group; $570.4 \pm 35.3$ for the debris + LPS/OEC group; $698.3 \pm 86.2$ for the LPS/OEC group; $730.5 \pm 183.2$ for the CCM/OEC group; $955 \pm 73.5$ for the debris $+\mathrm{LPS} /$ CCM/OEC group; and $1105.2 \pm 45.8$ for the LPS/CCM/ OEC group. Statistical analysis showed that degenerated neural debris can significantly inhibit neurite outgrowth and arborization, and the addition of LPS alone or CCM alone did not attenuate the inhibitory effect. When LPS alone or CCM alone was administered to the co-culture with OECs, it seemed that the inhibitory effects on neurite outgrowth were ameliorated, displaying longer neurite extensions and more process arborizations. As expected, a significant difference was found when compared with the normal and control groups. As for the combination of LPS and CCM in co-culture with OECs groups, LPS and CCM alone can remarkably promote neurite outgrowth and branching, and attenuate the inhibitory effects caused by degraded cell debris. Statistically, there was a significant difference compared with LPS-alone and CCMalone groups.

\section{The Possible Mechanism Underlying the Enhancement of Neuronal Growth Mediated by Activated OECs}

\section{Effect of CCM + LPS on Expression of TG2 and PSR in OECs}

Emerging evidence demonstrates that TG2 expression plays an important role in mediating numerous cellular activities such as binding extracellular matrix fibronectin, cell adhesion and motility, morphologic changes, and activating intracellular signaling pathways like phosphoinositide 3-kinase/protein kinase $\mathrm{B}$, glycogen synthase kinase- $3 \beta$, and nuclear factor kappa $\mathrm{B}(\mathrm{NF}-\mathrm{kB})$, which can regulate cell migration, cell proliferation, and cell survival [54-61]. In addition, PSR, an important receptor in recognizing a surface marker on apoptotic cells and initiating engulfment, can effectively mediate clearance of apoptotic cells, maintain normal tissue homeostasis, and play a role in regulation of the immune response [62-65]. Therefore, we examined the effects of LPS and CCM on TG2 and PSR expression in OECs using quantitative PCR, immunostaining, and Western blots. First, TG2 and PSR mRNA levels were examined in OECs treated under different conditions. After 1 and 3 days of treatment, OECs showed an increased level of TG2 (CCM alone) and a relatively higher level of PSR (LPS alone) when compared with normal OECs (Fig. 6a, b). Strikingly, the combination of LPS + CCM significantly elevated TG2 and PSR gene expression; however, there was no significant increase in TG2 mRNA at day 1. Next, we examined the expression of both molecules, using immunofluorescence. Representative photographs of TG2 staining are shown in Fig. 6c. No TG2 immunoreactivity was detectable in normal OECs. Moderate-to-high TG2 immunoreactivity was noted in OECs treated with LPS alone or CCM alone, and all remaining OECs had absent, weak, or intense immunoreactivity for TG2. When OECs were treated with the combination of LPS + CCM, almost all cells were strongly TG2-positive. Moreover, most cell populations exhibited more pronounced TG2 immunoreactivity when compared with LPS- or CCM-treated cells. Similar to TG2 immunofluorescence, PSR showed more pronounced immunoreactivity in the OECs treated with LPS and CCM than in those treated under other conditions (Fig. 6d). To further substantiate our findings, Western blot analysis was used to determine expression of both molecules. Consistent with the results of quantitative PCR and immunostaining, the combination of LPS + CCM led to a marked increase in either TG2 or PSR protein levels. Moreover, the increase in TG2 or PSR expression levels was time-dependent within 3 days (Fig. 6e).

\section{Disruption of TG2 and PSR Suppresses CCM- and LPS-Mediated OEC Activation}

To further substantiate if TG2 and PSR are predominantly involved in the CCM- and LPS-mediated OEC activation responsible for the enhancement of neuron outgrowth, OECs were pretreated with Cyst and NE prior to CCM and LPS constant co-stimulation. After 3 days of treatment, Cyst and NE significantly reduced CCM- and LPS-induced OEC proliferation, as demonstrated by the decreased percentage of $\mathrm{BrdU}^{+}$cells from $35.5 \% \pm 6.7 \%$ to $17.6 \% \pm 8.7 \%$ $(p<0.05)$. Strikingly, there was no significant difference in cell proliferation between normal and Cyst- and NE-treated in the presence or absence of CCM and LPS. Also, the percentage of OEC proliferation was much lower after Cyst and $\mathrm{NE}$ pretreatment than after CCM + LPS treatment only $(p<0.01)$ (Fig. 7a). Next, the assessment of phagocytic activity revealed that Cyst and NE significantly suppressed OEC ingestion of degenerated neural debris in the presence or $a b-$ sence of CCM and LPS, displaying a dramatically reduced amount of neural debris within OEC bodies (Fig. 7b). Consistently, quantitative analysis of the amount of engulfed debris by OECs also showed that Cyst and NE remarkably attenuated OEC phagocytic activity (Fig. 7c). Nonetheless, it is still not clear if the suppression of OEC properties by Cyst and NE could affect neuron outgrowth in the presence of degenerated neural debris. We further observed neuron 


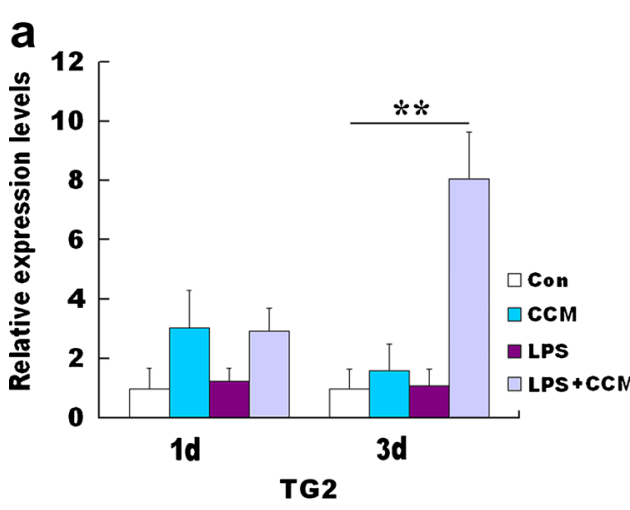

C
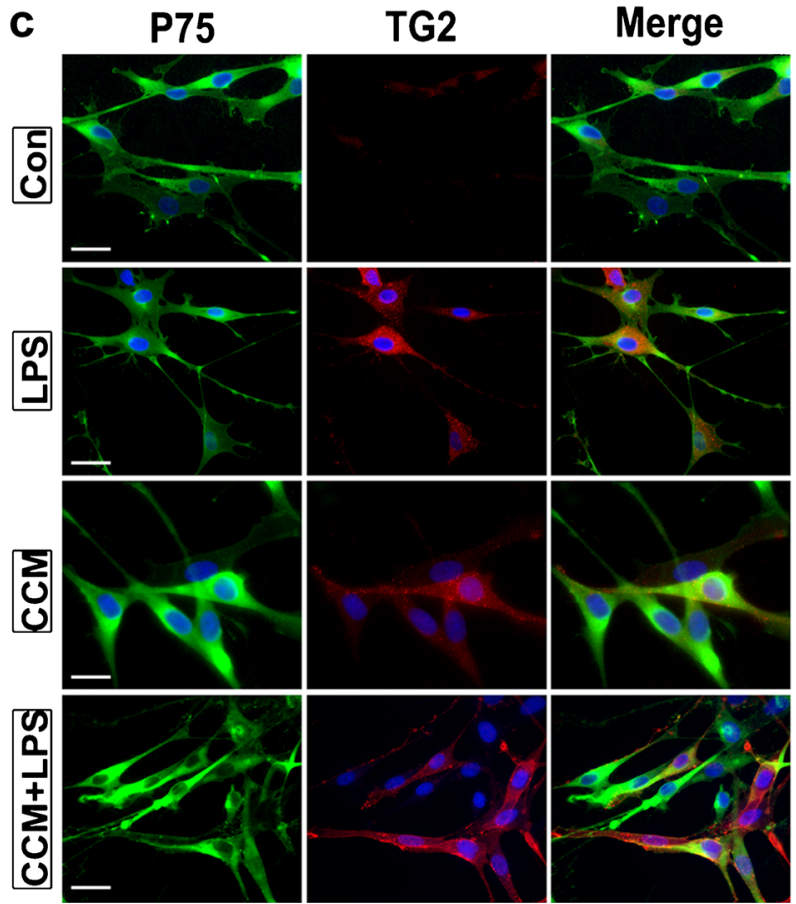

Fig. 6 Activation of signaling molecules is necessary for ingestion and phagocytosis of OECs. a, b Quantitative real-time polymerase chain reaction analysis of genes essential for olfactory ensheathing cell (OEC) phagocytosis under indicated treatments at 1 and 3 days. c, d Immunofluorescence revealed expression of transglutamase 2 (TG2) and phosphatidylserine receptor (PSR), respectively, in p75-positive cells treated under indicated conditions. e Western blot analysis of TG2

survival and neurite outgrowth when co-cultured with OECs pretreated with Cyst and NE prior to exposure of CCM and LPS. No difference in the number of surviving neurons and neurite extension was found between pretreatment Cyst and NE in the presence or absence of CCM and LPS groups; however, the number of surviving neurons in co-culture with OECs pretreated with Cyst and NE was significantly lower than those treated with CCM and LPS only. With regard to the no OEC groups, the administration of Cyst and NE aggravated the injury to neurons caused by degenerated neural debris, displaying a decrease in surviving neurons and more scattering of the degenerated debris in culture (Fig. 7d). These results e

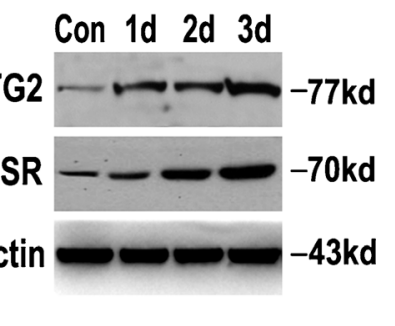

PSR
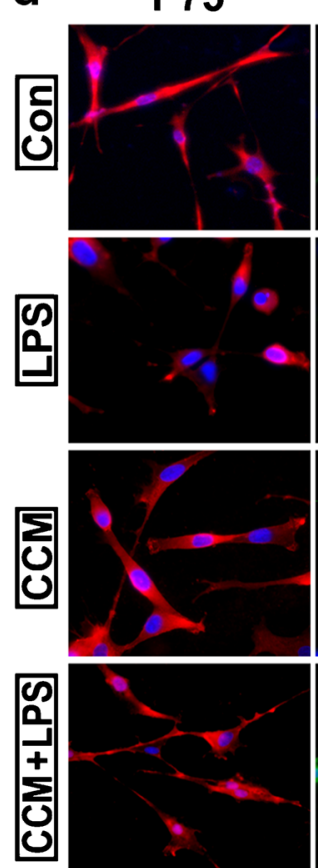
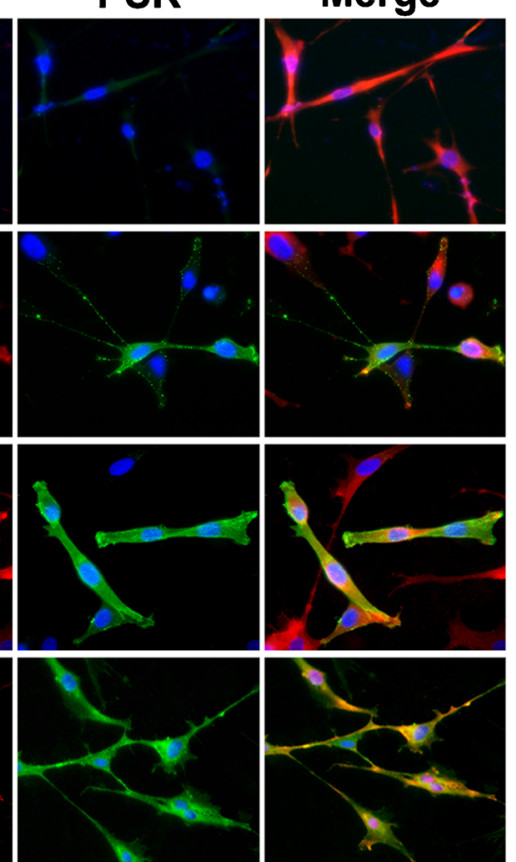

and PSR expression in OECs under indicated conditions for 1, 2, and 3 days. $\beta$-Actin served as loading controls. Notably, TG2 and PSR expression levels in OEC cytoplasm increased with prolongation of culture time. All data are reported as means \pm SEM. ${ }^{*} p<0.05$; $* * p<0.01, * * * p<0.01 v s$ corresponding controls (Con), analysis of variance post-hoc test. CCM = curcumin; LPS = lipopolysaccharide. Scale bars $=25 \mu \mathrm{m}$

implicated that TG2 and PSR strengthen CCM- and LPSmediated activation of OECs, which could contribute to neuron growth. Moreover, we quantified neuronal survival and neurite outgrowth. As shown in Fig. 7e, f, Cyst and NE dramatically suppressed the survival and neurite outgrowth of neurons mediated by CCM and LPS when co-cultured with OECs in the presence or absence of degenerated neural debris. The number of surviving neurons significantly decreased compared with the corresponding controls $(p<0.05$ or $p<0.01$ ). For normal neurons exposed to Cyst and NE, no remarkable change in the number of surviving neurons was found. Consistent with neuron survival, neurite growth was 


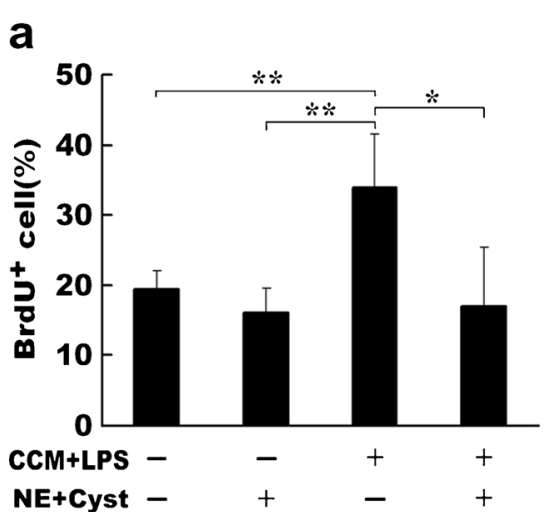

d
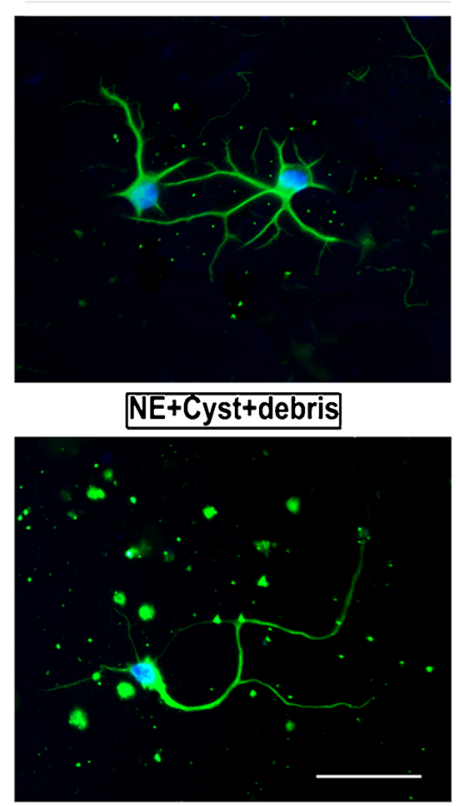

e

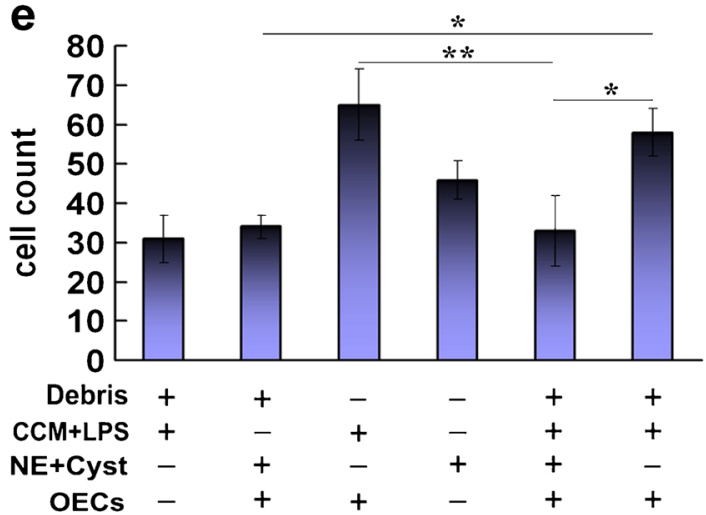

Fig. 7 Disruption of transglutamase 2 (TG2) and phosphatidylserine receptor (PSR) suppresses olfactory ensheathing cell (OEC) activation and phagocytic activity. a BrdU immunostaining revealed the proliferative capacity of OECs pretreated with cystamine (Cyst; inhibitor of TG2) and neutrophil elastase (NE; a cleavage enzyme of PSR) prior to curcumin (CCM) and lipopolysaccharide (LPS) constant co-stimulation. b Phase-contrast photographs of engulfed degenerated neural debris by OECs treated under the indicated conditions (red signals represent neural debris generated from red fluorescent protein

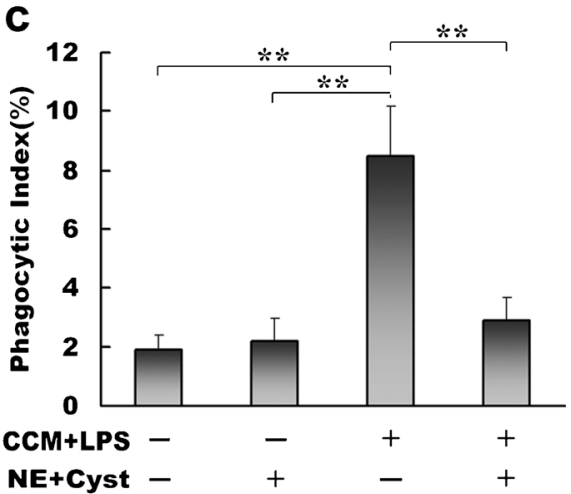

OECS+CCM+LPS

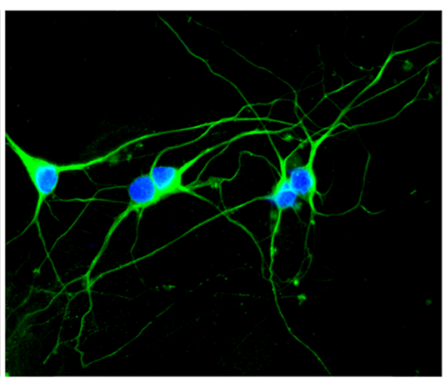

OECs+CCM+LPS+debris
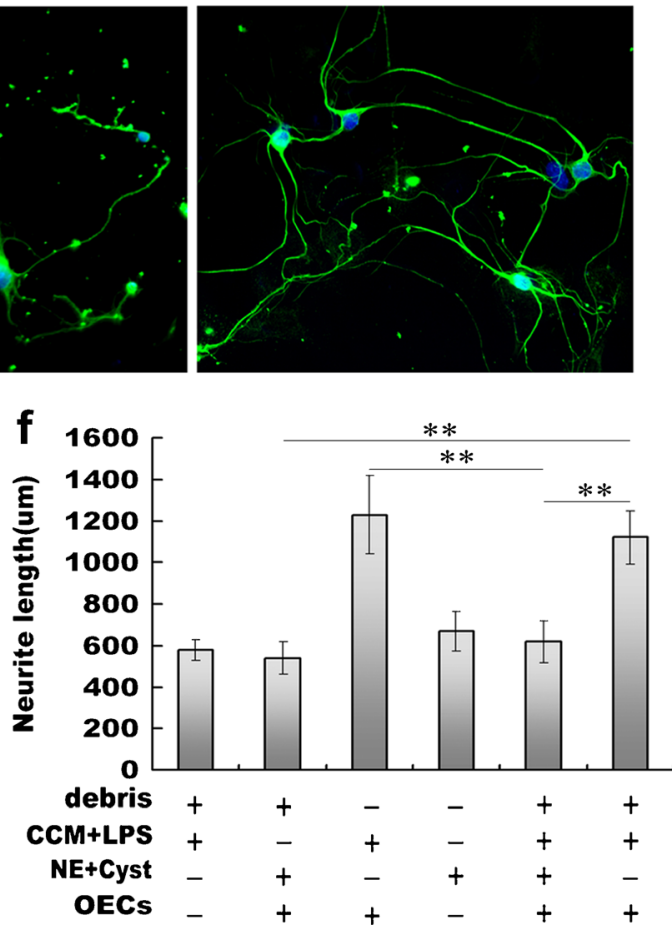

mouse). c Quantitative analysis of the relative amount of engulfed debris by OECs under the indicated treatments. d Representative images of primary spinal cord neuron at low density when co-cultured with OECs treated under the indicated conditions. Of note, green represents Tuj-1-positive staining. e, d Quantitative assessment of neuron survival and neurite length under indicated treatments, respectively. $* p<0.05 ; * * P<0.01$ vs the corresponding controls, analysis of variance post-hoc test. Scale bars $=200 \mu \mathrm{m}$ 
also inhibited by the administration of Cyst and NE. As expected, a significant difference was found when compared with the corresponding controls $(p<0.01$ or $p<0.001$; Fig. 7f).

\section{Discussion}

Currently, OECs are considered as attractive candidates for cell-based therapies for CNS injuries and neurodegenerative diseases. However, their proregeneration remains controversial. Some reports show no therapeutic efficacy for CNS insults, while a tremendous body of research states that OECs only mediate limited regeneration and functional recovery. Different laboratories show disparate results, ranging from little to extensive functional recovery $[12,13,66]$. Nonetheless, little is known about transplanted OECs in the damaged CNS sites. This is based on the fact that severe insults to CNS usually result in the presence of an unfavorable or hostile microenvironment in the injured zone. This hostile environment not only prevents neural regeneration, but also has a direct impact on transplanted OEC bioactivities, including survival, migration, proliferation, secretion, and phagocytosis [67]. In general, different lesion models induce different degrees of tissue damage and inflammation. The hostile and inhibitory environment arising from certain acute CNS damage may lead to the progressive death of numerous transplanted OECs, finally resulting in abortive or unsatisfactory neuroregeneration outcomes. Therefore, seeking an ideal and effective strategy for strengthening and releasing the therapeutic potential of OECs for eliciting neural regeneration by cell-based transplantation is of pivotal importance. Until now, little is known about enhancing the potential of OECs in creating a permissive environment as early as possible to repair CNS injuries and disorders.

In this study, we present in vitro data emphasizing the potential efficacy of a combination of LPS and CCM in inducing activation of OECs. Our results demonstrated convincingly that OECs can be significantly activated in conjunction with LPS and CCM. These activated OECs express a set of relative genes and proteins that define cell activation (CXCL1, CXCL2, TLR4, and TNF $\alpha)[9,31]$, and are endowed with enhanced proliferation and phagocytic capacity. More importantly, activated OECs can efficiently promote neural survival and neurite outgrowth in the presence of degenerated neuronal debris. In comparison with other treatment conditions (LPS alone, CCM alone), OECs treated with a combination of LPS and the CCM acquired the most pronounced capacity to promote neuronal growth. Moreover, the elevated beneficial activation of OECs may be intimately associated with upregulation of TG2 and PSR. Therefore, the present therapeutic approach using activated OECs could be a promising option for potential cell therapies targeting acute CNS injury and neurodegenerative diseases.

As CNS injuries or neurodegenerative disorders occur, a significant proportion of neurons will undergo a complicated degeneration process, resulting in large amounts of harmful substances, for example cell debris and inhibitory molecules, in the injury lesions $[8,9,17]$. Therefore, rapid and efficient clearance of degenerated products is required for neuronal survival and neurite growth. Our recent study has shown that OECs have the capacity to ingest and engulf degenerated nerve tissue debris, resulting in neuron growth [8]. Despite this, formation of a local hostile environment after CNS damage does not usually support the survival of OECs, even though OECs have strong adaptability. Thus, they could not be used to promote neural regeneration. Fortunately, the proliferation and phagocytosis capacity of OECs can be markedly strengthened after activation. This can be used to remedy progressive OEC death and further deterioration of the microenvironment within injured neural tissue, but is dependent on continuous proliferation of endogenous OECs to further replenish the loss of transplanted OECs without the need for secondary transplantation. On the basis of these elucidations, we wished to induce OEC activation with a combination of LPS and CCM. Our data proved that the combined treatment can effectively induce the activation of OECs, indicating that this protocol is of particular interest for neural repair therapies.

It was reported previously that LPS can lead to functional activation of OECs $[9,68]$, by improving their phagocytic capacity. These findings are in line with our recent reports, except that no distinct promotion of neuron survival and neurite outgrowth in co-culture with LPS-treated OECs was found in our experiments. Although rapid and efficient clearance of degenerated cell debris is of importance for neural regeneration, limited and slow removal of debris after CNS injury seems less supportive. Retention of degenerated cell debris can trigger a serious of inflammatory cascades, which are harmful to cells as a result of the release of oxygen free radicals and neurotoxic enzymes [21, 69, 70]. Therefore, OEC proliferation, motility, and migration are crucial for creating a favorable environment for both nerve regeneration and restoration. CCM has been shown to stimulate the proliferation of neural progenitors [46]. More recently, another report has demonstrated that CCM selectively regulates the activation of extracellular-regulated kinase and p38 mitogen-activated protein kinase pathways to elicit OEC proliferation and migration [45]. In our study, we found that CCM effectively improved OEC proliferation and phagocytic capacity in presence of LPS. Intriguingly, further exploration revealed that OEC activation induced by the combination of LPS and CCM remarkably promoted neuron survival and neurite outgrowth under hostile conditions (delivering a considerable amount of degenerated cell debris). This positive effect may associate with a combination of OEC proliferation, motility, 
and migration. First, OEC proliferation results in an increase in cell numbers, which effectively decreases the amount of debris in culture, replenishes the loss of OECs in the hostile environment, and elevates total secretion of neurotrophic molecules. Second, motility and migration of OECs may be improved [46], which further supports better phagocytosis. Notwithstanding, LPS alone or CCM alone seems to weakly promote OEC activation, resulting in moderate levels of proliferation and phagocytosis. Of note, these promotive effects are inferior to the combination of LPS and CCM, suggesting that LPS and CCM might exert a synergetic effect in inducing OEC activation.

In the present study, LPS was used to enhance the activation of OECs, which may lead to some risks in future clinical translation because LPS can cause neuroinflammation by triggering activation of microglial cells in vivo. The neuroinflammation may further exacerbate neuronal damage. Nevertheless, the neuroinflammation arising from activating microglial cells in vivo must be dependent on high-dose LPS stimulation, while the low dose of LPS used in our study could hardly evoke effective activation of microglial cells in vivo. In comparison, the rapid removal of degenerated debris by activated OECs may be more important in improving the microenvironment, reducing inflammatory injury and further promoting neuronal survival and regeneration. Even though LPS at $1 \mu \mathrm{M}$ may cause slight activation of microglia in vivo and further result in neuroinflammation at a low level, the inflammation may only result in negligible negative effect on neurons. In addition, CCM has a significant anti-inflammatory effect $[42,43]$, which may offset the hazard caused by the LPS-induced minor inflammatory reaction.

In order to further substantiate our hypothesis, TG2 and PSR expression in OECs treated with LPS and CCM were investigated in several assays. The results revealed that the combination of LPS and CCM elevated TG2 and PSR remarkably, both in regard to gene transcription and protein expression levels, suggesting that the combination of LPS and CCM truly elicits OEC activation because TG2 and PSR, as either mediator or effector in their downstream signaling, were activated. In line with our results, it has been demonstrated that TG2 plays an important role in activating an intracellular signaling cascade and receptor signaling that are tightly associated with cell proliferation and motility [54, 58, 61]. Moreover, TG2 can mediate NF- $\mathrm{KB}$ activation, resulting in Akt activation $[57,58]$. It is well-known that NF- $\kappa B$ activation can facilitate cell survival and cell proliferation [44, 46, $61,71]$. Recent studies have demonstrated that upregulation of TG2 in epithelial cells results in constructive activation of focal adhesion kinase, Akt, NF- $\mathrm{BB}$, RhoA, and mitogenactivated protein kinase, contributing to cell adhesion, migration, and invasion [53, 54, 56, 72, 73]. Accordingly, the possible underlying mechanism of OEC activation and subsequent promotion of neuron growth is associated with upregulation of TG2 caused by synergistic stimulation by LPS and CCM. Meantime, TG2 upregulation is a likely explanation of the abovementioned cellular events. Strikingly, our data also revealed that PSR, a critical mediator in phagocytosis, was significantly upregulated at the mRNA and protein levels. Normally, PSR is displayed on the outer of membrane leaflet as a so-called "eat-me" signal [64]. When apoptosis and necrosis occur, cell surface-exposed PSR usually serves as a single and direct signal for recognition/uptake by phagocytic cells $[62,63]$. Increased PSR expression levels are indicative of increased phagocytic activity [9, 65]. As expected, we also observed that LPS and CCM can significantly increase the expression of PSR when compared with that induced by LPS alone, CCM alone, or normal control, further emphasizing the synergistic effects of LPS and CCM on OEC activation and phagocytosis. On the basis of our present data, as well as on several previous reports, upregulation of TG2 and PSR may be involved in LPS- and CCM-mediated activation of OECs and the promoting effects on neurons. Still, it is unclear whether the signaling of TG2 and PSR is truly responsible for possible mechanisms underlying the special cell event. In order to elucidate the issue, Cyst (a specific inhibitor of TG2) and NE (a cleavage enzyme of PSR [74]) were administered into OEC cultures/neuron and OEC cocultures pretreated with LPS and CCM. Our results revealed that disruption of TG2 and PSR biofunctions effectively abrogated OEC activation and the further promotive effects on neurons, implying that the signaling of TG2 and PRS is involved in LPS- and CCM-mediated cellular events. Although the precise mechanisms responsible for the activation of OECs induced by LPS and CCM and subsequent promotive effects on neuron growth remain unclear, a possible mechanism modulated by LPS and CCM eliciting TG2 and PRS upregulation by OECs could account for the intricate event.

\section{Conclusions}

We conclude that the combination of LPS and CCM can remarkably potentiate activation of OECs, resulting in a strengthened phagocytic capacity of OECs with regard to degenerated debris, which, in turn, efficiently promotes neuron survival and neurite outgrowth. Furthermore, we also unravel the possible molecular mechanisms responsible for the cascade event. This study is likely to provide a novel strategy for autologous OECbased transplantation in CNS injury and a variety of neurological disorders. Furthermore, we clarify controversial issues regarding OEC biofunctions in proregeneration.

Acknowledgments This work was supported by the Natural Science Foundation of China (grants: 81371411, 81472098, 81571208, 81670846 , and YJ2014001). The authors confirm that there has been no financial support for this research that could have influenced its outcome. 
Required Author Forms Disclosure forms provided by the authors are available with the online version of this article.

\section{References}

1. Woodhall E, West AK, Chuah MI. Cultured olfactory ensheathing cells express nerve growth factor, brain-derived neurotrophic factor, glia cell line-derived neurotrophic factor and their receptors. Brain Res Mol Brain Res 2001;88:203-213.

2. Chung RS, Woodhouse A, Fung S, et al. Olfactory ensheathing cells promote neurite sprouting of injured axons in vitro by direct cellular contact and secretion of soluble factors. Cell Mol Life Sci 2004;61:1238-1245

3. Pastrana E, Moreno-Flores MT, Avila J, Wandosell F, Minichiello L, Diaz-Nido J. BDNF production by olfactory ensheathing cells contributes to axonal regeneration of cultured adult CNS neurons. Neurochem Int 2007;50:491-8.

4. Roet KC, Verhaagen J. Understanding the neural repair-promoting properties of olfactory ensheathing cells. Exp Neurol 2014;261: 594-609

5. Lipson AC, Widenfalk J, Lindqvist E, Ebendal T, Olson L. Neurotrophic properties of olfactory ensheathing glia. Exp Neurol 2003; $180: 167-71$

6. Witheford M, Westendorf K, Roskams AJ. Olfactory ensheathing cells promote corticospinal axonal outgrowth by a L1 CAMdependent mechanism. Glia 2013;61:1873-89.

7. Srivastava N, Seth K, Khanna VK, Ansari RW, Agrawal AK. Longterm functional restoration by neural progenitor cell transplantation in rat model of cognitive dysfunction: co-transplantation with olfactory ensheathing cells for neurotrophic factor support. Int J Dev Neurosci 2009;27:103-110.

8. He BR, Xie ST, Wu MM, Hao DJ, Yang H. Phagocytic removal of neuronal debris by olfactory ensheathing cells enhances neuronal survival and neurite outgrowth via p38MAPK activity. Mol Neurobiol 2014;49:1501-1512.

9. Su Z, Chen J, Qiu Y, et al. Olfactory ensheathing cells: the primary innate immunocytes in the olfactory pathway to engulf apoptotic olfactory nerve debris. Glia 2013;61:490-50.

10. Wanner IB, Deik A, Torres M, et al. A new in vitro model of glial scar inhibits axon growth. Glia 2008;56:1691-1709

11. Wanner IB, Anderson MA, Song B, et al. Glial scar borders are formed by newly proliferated, elongated astrocytes that interact to corral inflammatory and fibrotic cells via STAT3-dependent mechanisms after spinal injury. J Neurosci 2013;33:12870-12886

12. Riddell JS, Enriquez-Denton M, Toft A, Fairless R, Barnett SC. Olfactory ensheathing cell grafts have minimal influence on regeneration at the dorsal root entry zone following rhizotomy Glia 2004; 47:150-167.

13. Lu P, Yang H, Culbertson M, Graham L, Roskams AJ, Tuszynski $\mathrm{MH}$. Olfactory ensheathing cells do not exhibit unique migratory or axonal growth-promoting properties after spinal cord injury. J Neurosci 2006;26:11120-11130

14. Mukhopadhyay G, Doherty P, Walsh FS, Crocker PR, Filbin MT. A novel role for myelin-associated glycoprotein as an inhibitor of axonal regeneration. Neuron 1994;13:757-767.

15. Chen MS, Huber AB, van der Haar ME, et al. Nogo-A is a myelin associated neurite outgrowth inhibitor and an antigen for monoclonal antibody IN-1. Nature 2000;403:434-439

16. Wang KC, Koprivica V, Kim JA, et al. Oligodendrocyte-myelin glycoprotein is a Nogo receptor ligand that inhibits neurite outgrowth. Nature 2002;417:941-944
17. Hata K, Fujitani M, Yasuda Y, et al. RGMa inhibition promotes axonal growth and recovery after spinal cord injury. J Cell Biol 2006; $173: 47-58$

18. Tanaka T, Ueno M, Yamashita T. Engulfment of axon debris by microglia requires p38 MAPK activity. J Biol Chem 2009;284: 21626-21636

19. Hulsebosch CE. Recent advances in pathophysiology and treatment of spinal cord injury. Adv Physiol Educ 2002; 26:238-255

20. Li S, Liu BP, Budel S, et al. Blockade of Nogo-66, myelinassociated glycoprotein, and oligodendrocyte myelin glycoprotein by soluble Nogo-66 receptor promotes axonal sprouting and recovery after spinal injury. J Neurosci 2004; 24:10511-10520.

21. Fleming JC, Norenberg MD, Ramsay DA, et al. The cellular inflammatory response in human spinal cords after injury. Brain 2006; 129:3249-3269.

22. Lauber K, Blumenthal SG, Waibel M, Wesselborg S. Clearance of apoptotic cells: getting rid of the corpses. Mol Cell 2004; 14:277228

23. Panni P, Ferguson IA, Beacham I, Mackay-Sim A, Ekberg JA, St John JA. Phagocytosis of bacteria by olfactory ensheathing cells and Schwann cells. Neurosci Lett 2013; 539:65-70.

24. Nazareth L, Lineburg KE, Chuah MI, et al. Olfactory ensheathing cells are the main phagocytic cells that remove axon debris during early development of the olfactory system. J Comp Neurol 2015; 523:479-494

25. Nazareth L, Tello Velasquez J, Lineburg KE, Chehrehasa F, St John JA, Ekberg JA. Differing phagocytic capacities of accessory and main olfactory ensheathing cells and the implication for olfactory glia transplantation therapies. Mol Cell Neurosci 2015; 65:92-101

26. Leung JY, Chapman JA, Harris JA, et al. Olfactory ensheathing cells are attracted to, and can endocytose, bacteria. Cell Mol Life Sci 2008; 65:2732-2739

27. Kulp A, Kuehn MJ. Biological functions and biogenesis of secreted bacterial outer membrane vesicles. Annu Rev Microbiol 2010; 64: 163-184.

28. Mansoor UF, Vitharana D, Reddy PA, et al. Design and synthesis of potent Gram-negative specific LpxC inhibitors. Bioorg Med Chem Lett 2011;21:1155-1161

29. Tsatsanis C, Zacharioudaki V, Androulidaki A, et al. Adiponectin induces TNF-alpha and IL-6 in macrophages and promotes tolerance to itself and other pro-inflammatory stimuli. Biochem Biophys Res Commun 2005;335:1254-1263.

30. Gertsch J. Anti-inflammatory cannabinoids in diet: Towards a better understanding of $\mathrm{CB}(2)$ receptor action? Commun Integr Biol 2008; 1:26-28.

31. Vincent AJ, Taylor JM, Choi-Lundberg DL, West AK, Chuah MI. Genetic expression profile of olfactory ensheathing cells is distinct from that of Schwann cells and astrocytes. Glia 2005; 51:132-147

32. Yan H, Lu D, Rivkees SA. Lysophosphatidic acid regulates the proliferation and migration of olfactory ensheathing cells in vitro. Glia 2003; 44:26-36.

33. Hatcher H, Planalp R, Cho J, Tortia FM, Torti SV. Curcumin: From ancient medicine to current clinical trials. Cellular and Molecular Life Sci 2008; 65: 1631-1652

34. Vecchi Brumatti L, Marcuzzi A, Tricarico PM, Zanin V, Girardelli M, Bianco AM. Curcumin and inflammatory bowel disease: potential and limits of innovative treatments. Molecules 2014; 19: $21127-$ 21153.

35. Okudan N, Belviranlı M, Gökbel H, Oz M, Kumak A. Protective effects of curcumin supplementation on intestinal ischemia reperfusion injury. Phytomedicine 2013; 20:844-848

36. Cox KH, Pipingas A, Scholey AB. Investigation of the effects of solid lipid curcumin on cognition and mood in a healthy older population. J Psychopharmacol 2015; 29: 642-651 
37. Kaur H, Patro I, Tikoo K, Sandhir R. Curcumin attenuates inflammatory response and cognitive deficits in experimental model of chronic epilepsy. Neurochem Int 2015; 89:40-50

38. Huang TY, Tsai TH, Hsu CW, Hsu YC. Curcuminoids suppress the growth and induce apoptosis through caspase-3-dependent pathways in glioblastoma multiforme (GBM) 8401 cells. J Agric Food Chem 2010; 58:10639-10645.

39. Hoppe JB, Coradini K, Frozza RL, et al. Free and nanoencapsulated curcumin suppress $\beta$-amyloid-induced cognitive impairments in rats: involvement of BDNF and Akt/GSK-3 $\beta$ signaling pathway. Neurobiol Learn Mem 2013; 106:134-144.

40. Pae HO, Jeong SO, Zheng M, et al. Curcumin attenuates ethanolinduced toxicity in HT22 hippocampal cells by activating mitogenactivated protein kinase phosphatase-1. Neurosci Lett 2009; 453: 186-189

41. Onder A, Kapan M, Gumus M, et al. The protective effects of curcumin on intestine and remote organs against mesenteric ischemia/reperfusion injury. Turk J Gastroenterol 2012; 23: 141-147

42. Mandal MN, Patlolla JM, Zheng L, et al. Curcumin protects retinal cells from light-and oxidant stress-induced cell death. Free Radic Biol Med 2009; 46: 672-679

43. Kelsey NA, Wilkins HM, Linseman DA. Nutraceutical antioxidants as novel neuroprotective agents. Molecules 2010; 15:7792-7814.

44. Wang YF, Zu JN, Li J, Chen C, Xi CY, Yan JL. Curcumin promotes the spinal cord repair via inhibition of glial scar formation and inflammation. Neurosci Lett 2014; 560:51-56.

45. Tello Velasquez J, Watts ME, Todorovic M, et al. Low-dose curcumin stimulates proliferation, migration and phagocytic activity of olfactory ensheathing cells. PLOS ONE 2014; 9: e111787

46. Kim SJ, Son TG, Park HR, et al. Curcumin stimulates proliferation of embryonic neural progenitor cells and neurogenesis in the adult hippocampus. J Biol Chem 2008; 283: 14497-14505

47. Tello Velasquez J, Nazareth L, Quinn RJ, Ekberg JA, St John JA. Stimulating the proliferation, migration and lamellipodia of Schwann cells using low-dose curcumin. Neuroscience 2016; 2: 324:140-150

48. Liao KK, Wu MJ, Chen PY, et al. Curcuminoids promote neurite outgrowth in PC12 cells through MAPK/ERK- and PKCdependent pathways. J Agric Food Chem 2012; $60: 433-443$

49. Yao M, Yang L, Wang J, et al. Neurological recovery and antioxidant effects of curcumin for spinal cord injury in the rat: a network metaanalysis and systematic review. J Neurotrauma 2015; 32:381-391

50. Ramón-Cueto A, Cordero MI, Santos-Benito FF, Avila J. Functional recovery of paraplegic rats and motor axon regeneration in their spinal cords by olfactory ensheathing glia. Neuron 2000;25: 425-435

51. Yang H, Cheng X, Yao Q, Li J, Ju G. The promotive effects of thymosin beta4 on neuronal survival and neurite outgrowth by upregulating L1 expression. Neurochem Res 2008; 33:2269-2280.

52. Yang H, Cheng XP, Li JW, Yao Q, Ju G. De-differentiation response of cultured astrocytes to injury induced by scratch or conditioned culture medium of scratch-insulted astrocytes. Cell Mol Neurobiol 2009; 29: 455-473.

53. Sergent-Tanguy S, Chagneau C, Neveu I, Naveilhan P. Fluorescent activated cell sorting (FACS): a rapid and reliable method to estimate the number of neurons in a mixed population. J Neurosci Methods 2003; 129:73-79.

54. Akimov SS, Belkin AM. Cell surface tissue transglutaminase is involved in adhesion and migration of monocytic cells on fibronectin. Blood 2001;98:1567-1567

55. Akimov SS, Belkin AM. Cell-surface transglutaminase promotes fibronectin assembly via interaction with the gelatin-binding domain of fibronectin: a role in TGFbeta-dependent matrix deposition. J Cell Sci 2001; 114:2989-3000.
56. Lee J, Kim YS, Choi DH, et al. Transglutaminase-2 induces nuclear factor-kappaB activation via a novel pathway in BV-2 microglia. J Biol Chem 2004; 279:53725-54735

57. Kang SK, Lee JY, Chung TW, Kim CH. Overexpression of transglutaminase-2 accelerates the erythroid differentiation of human chronic myelogenous leukemia K562 cell line through PI3K/Akt signaling pathway. FEBS Lett 2004; 577:361-366

58. Wang Y, Ande SR, Mishra S. Phosphorylation of transglutaminase 2 (TG2) at serine-216 has a role in TG2 mediated activation of nuclear factor-kappa B and in the downregulation of PTEN. BMC Cancer 2012;12:277

59. Boroughs LK, Antonyak MA, Cerione RA. A novel mechanism by which tissue transglutaminase activates signaling events that promote cell survival. J Biol Chem 2014; 289:10115-10125

60. Almami I, Dickenson JM, Hargreaves AJ, Bonner PL. Modulation of transglutaminase 2 activity in $\mathrm{H} 9 \mathrm{c} 2$ cells by PKC and PKA signalling: a role for transglutaminase 2 in cytoprotection.Br J Pharmacol 2014; 171:3946-3960.

61. Eckert RL, Kaartinen MT, Nurminskaya M, et al. Transglutaminase regulation of cell function. Physiol Rev 2014; 94:383-417.

62. Fadok VA, Bratton DL, Rose DM, Pearson A, Ezekewitz RA, Henson PM. A receptor for phosphatidylserine-specific clearance of apoptotic cells. Nature. 2000; 405: 85-90.

63. Li MO, Sarkisian MR, Mehal WZ, Rakic P, Flavell RA. Phosphatidylserine receptor is required for clearance of apoptotic cells. Science 2003;302:1560-1563

64. Wong K, Valdez PA, Tan C, Yeh S, Hongo JA, Ouyang W. Phosphatidylserine receptor Tim-4 is essential for the maintenance of the homeostatic state of resident peritoneal macrophages. Proc Natl Acad Sci U S A 2010;107:8712-8717

65. Yang H, Chen YZ, Zhang Y, et al. A lysine-rich motif in the phosphatidylserine receptor PSR-1 mediates recognition and removal of apoptotic cells. Nat Commun 2015; 6:5717

66. Lee IH, Bulte JW, Schweinhardt P, et al. In vivo magnetic resonance tracking of olfactory ensheathing glia grafted into the rat spinal cord. Exp Neurol 2004;187:509-516

67. Barnett SC, Riddell JS. Olfactory ensheathing cell transplantation as a strategy for spinal cord repair - what can it achieve? Nat Clin Pract Neurol 2007; 3:152-161.

68. Vincent AJ, Choi-Lundberg DL, Harris JA, West AK, Chuah MI. Bacteria and PAMPs activate nuclear factor kappaB and Gro production in a subset of olfactory ensheathing cells and astrocytes but not in Schwann cells. Glia 2007; 55:905-916.

69. Hassler SN, Johnson KM, Hulsebosch CE. Reactive oxygen species and lipid peroxidation inhibitors reduce mechanical sensitivity in a chronic neuropathic pain model of spinal cord injury in rats. $\mathbf{J}$ Neurochem 2014; 131:413-417.

70. Cooney SJ, Zhao Y, Byrnes KR. Characterization of the expression and inflammatory activity of NADPH oxidase after spinal cord injury. Free Radic Res 2014; 48:929-939

71. Siegel M, Khosia C. Transglutaminase 2 inhibitors and their therapeutic role in disease states. Pharmacol Ther 2007; 115:232-245

72. Tong L, Png E, Aihua H, et al. Molecular mechanism of transglutaminase-2 in corneal epithelial migration and adhesion. Biochim Biophys Acta 2013;1833:1304-1315

73. Janiak A, Zemskov EA, Belkin M. Cell surface transglutaminase promotes RhoA activation via integrin clustering and suppression of the Src-p190RhoGAP signaling pathway. Mol Biol Cell 2006; 17:1606-1619

74. Vandivier RW, Fadok VA, Hoffmann PR, et al. Elastase-mediated phosphatidylserine receptor cleavage impairs apoptotic cell clearance in cystic fibrosis and bronchiectasis. J Clin Invest 2002; 109: 661-670 